\title{
Regression as the Univariate General Linear Model: Examining Test Statistics, p values, Effect Sizes, and Descriptive Statistics Using R
}

\begin{tabular}{lccc}
\hline Kim Nimon & Julia Berrios & $\begin{array}{c}\text { Greggory L. Keiffer } \\
\text { Mandolen Mull }\end{array}$ & Jon Musgrave \\
& University of Texas at Tyler & \\
\hline \hline
\end{tabular}

This paper presents regression as the univariate general linear model (GLM). Building on the work of Cohen (1968), McNeil (1974), and Zientek and Thompson (2009), the paper uses descriptive statistics to build a small, simulated dataset that readers can use to verify that multiple linear regression (MLR) subsumes the univariate parametric analyses in the GLM. Unlike other related works, we provide R syntax that demonstrates how MLR produces equivalent test statistics, $p$ values, effect sizes, and descriptive statistics when compared to the univariate analyses that MLR subsumes. The paper diverges from Zientek and Thompson by presenting an expanded hierarchy for MLR and demonstrating why only the case of the chi-square test of independence where the criterion variable is dichotomous, and not the general case, is subsumed by MLR. Readers will find an accessible treatment of the GLM as well as R syntax, which they can use to report descriptive statistics, $p$ values, and effect sizes associated with the univariate parametric statistics in the GLM.

$\mathrm{I}$ n 1968, Cohen presented multiple linear regression (MLR) as the univariate general linear model (GLM). Since that time, Cohen's work has been extended to consider canonical correlation as the multivariate GLM (see Knapp, 1978) and structural equation modeling as an even more general case of the GLM (see Bagozzi, Fornell, \& Larcker, 1981). As noted by Graham (2008),

The vast majority of parametric statistical procedures in common use are part of [a single analytic family called] the General Linear Model (GLM), including the $t$ test, analysis of variance (ANOVA), multiple regression, descriptive discriminant analysis (DDA), multivariate analysis of variance (MANOVA), canonical correlation analysis (CCA), and structural equation modeling (SEM). Moreover, these procedures are hierarchical in that some procedures are special cases of others. (p. 485).

In addition to the hierarchical nature of the GLM is the concept that the subsumed analyses share three characteristics. Analyses in the GLM implicitly or explicitly are correlational in nature, yield variance accounted effect sizes, and produce scores on latent variables that are derived by applying weights to measured variables (Thompson, 2006, p. 360).

Although the characteristics of the GLM seem to be straightforward, graduate students and emerging scholars are likely to benefit from being able to verify the hierarchical nature of the GLM through illustrations that compare univariate statistical analyses to MLR analyses. Not only has active learning been shown to be beneficial when learning statistics (White, 2015), research (e.g., Henson, Hull, \& Williams, 2010) indicates that many graduate students and emerging scholars may have insufficient quantitative proficiency. Therefore, we offer an illustration of MLR as the univariate GLM that considers the similarities and differences in the test statistics, $p$ values, effect sizes, and descriptive statistics generated. Namely, we consider ANCOVA, ANOVA, $r$, repeated measures ANOVA (RM ANOVA), independent samples $t$-test, paired-samples $t$-test, and single-sample $t$-test. Our interest in developing this work is similar to other methodologists who seek to "improve statistical practice, and thereby, improve the quality of the knowledge produced by the legions of researchers around the world who use these techniques on a daily basis" (Osborne, 2013, p. 1).

We also make five unique contributions to the literature. We demonstrate MLR as the univariate GLM for parametric analyses using $\mathrm{R}$, which is a free statistical programming language that is gaining popularity in social science research and that is compatible with Unix, Windows, and Mac operating systems (R Development Core Team, 2017). Prior contributions (e.g., Zientek \& Thompson, 2009) have used commercial statistical software packages (e.g., SPSS). Second, we demonstrate that the hierarchical 


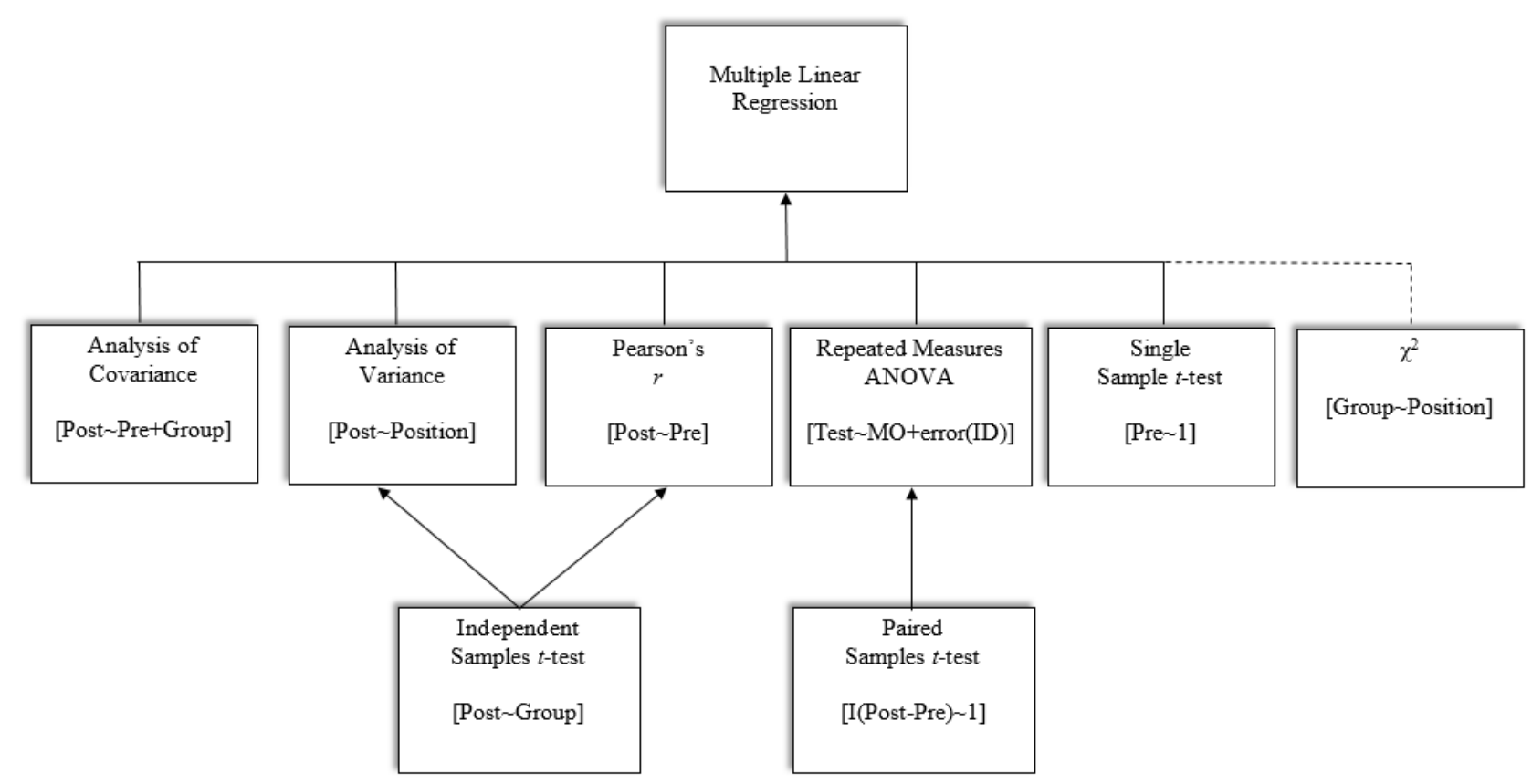

Figure 1. Multiple linear regression (MLR) as the univariate general linear model. Dotted line indicates that $\chi^{2}$ test of independence is only assumed by MLR in the case of a dichotomous dependent variable. Illustrative models identified in [ ]. See formula in stats package (R Development Core Team, 2017) for formatting of model formulae.

nature of the univariate parametric statistical analyses is not as flat as portrayed in Zientek and Thompson (p. 344). Namely, we show that ANOVA and $r$ subsume the independent samples $t$-test. Not only is it important to show that these analyses (i.e., ANOVA, $r$, and independent samples $t$-test) are mathematically equivalent, demonstrating that $r$ subsumes the independent samples $t$-test helps undo the misconception that correlation never implies causality and that causality is a function of design, not statistics (cf. Huck, 2012). Third, we demonstrate that RM ANOVA is subsumed by MLR and subsumes the paired-samples $t$-test. Fourth, we demonstrate that the single-sample $t$-test is subsumed by MLR. Finally, we demonstrate why the general case for the chi-square test of independence cannot be subsumed by MLR and that only in the case of a dichotomous dependent variable does MLR subsume the chi-square test. Therefore, the hierarchy of analyses subsumed by MLR presented in Figure 1, which serves as a framework for our paper, diverges from the hierarchy presented by Zientek and Thompson (p. 344) in important ways.

\section{Method}

The syntax in Appendix A was used to generate the datasets in Tables 1 and 2 that serve as the basis of the analyses illustrated. The dataset contains four variables: pretest scores (Pre), posttest scores (Post), follow-up scores (FollowUp), group assignment (Control, Treatment) and position (full-time [Full], parttime [Part], seasonal [Seasonal]). The dataset was designed so that each group has equal variances $(S D=$ 1) and equal covariances $(r s=0.6)$ between the pretest, posttest, and follow-up scores to satisfy statistical assumption in the illustrated analyses. In both groups, the mean pretest score is 4.0. In the control and treatment groups, the mean posttest score is, respectively, 4.0 and 6.0. In the control and treatment groups, the mean follow-up score is, respectively, 4.0 and 5.5. Table 1 was used as input to all of the analyses with the exception of the RM ANOVA analyses, where Table 2 was used. Table 1 is considered the wide representation of the data as each repeated measure (i.e., Pre, Post, and FollowUp) is represented in a separate column. Table 2 is considered the long representation of the data as the three repeated measures are contained in one column (Test), with a corresponding column that indicates the particular measurement occasion (MO), where 1,2, and 3, respectively, indicate pretest, posttest, and follow-up. 
ANCOVA. An ANCOVA was run with posttest scores, pretest scores, and group assignment, respectively, serving as the dependent, covariate, and independent variable. Pretest scores were centered at the group mean in order to have a meaningful intercept. A linear model with and without the covariate was analyzed and then compared with ANOVA to facilitate an ANCOVA analysis in R (cf. Crawley, 2013). In the MLR model, posttest scores were regressed on the pretest scores and the grouping variable. The ANCOVA models tested the hypothesis that group had a statistically and practically significant effect on posttest scores after controlling for pretest scores. Test statistics, $p$ values, effect sizes, and adjusted group means were compared between the results of the two analyses.

ANOVA. A MLR and ANOVA were run with posttest scores and position, respectively, serving as the dependent and independent variables. The ANOVA models tested the hypothesis that there was a statistically and practically significant difference in posttest scores by position. Test statistics, $p$ values, effect sizes, and group means were compared between the results of the two analyses.

$\boldsymbol{r}$. A MLR and $r$ were run with posttest and pretest scores, respectively, serving as the dependent and independent variables. The $r$ models tested the hypothesis that there was a statistically and practically significant relationship between posttest and pretest scores. Test statistics, $p$ values, and effect sizes were compared between the results of the two analyses.

RM ANOVA. A MLR and RM ANOVA were run testing the hypotheses that pretest, posttest, and followup scores are statistically and practically different. For MLR, test scores (see Table 2) were modeled by measurement occasion (i.e., 1=pretest, 2=posttest, $3=$ measurement occasion) and participant ID. For RM ANOVA, test scores were modeled by measurement occasion and individual error (cf. Fox \& Weisberg, 2011). measurement occasion means were compared between the results of the two analyses.

Independent Samples $t$-test. A MLR, ANOVA, $r$, and independent samples $t$-test were run with posttest scores and group, respectively, serving as the dependent and independent variables. The numeric representation of group served as the independent variable for $r$. The independent samples $t$-test models tested the hypotheses that there was a statistically and practically significant mean difference in posttest scores by group. Test statistics, $p$ values, effect sizes, and group means were compared among the results of the four analyses.

Paired-Samples $t$-test. A MLR, RM ANOVA, and paired-samples $t$-test were run testing the hypotheses that posttest scores are statistically and practically different than pretest scores. For MLR, the difference between posttest and pretest scores served as the dependent variable, which was modeled only by the intercept. For RM ANOVA, test scores were modeled by measurement occasion (i.e., 1=pretest, 
Table 2. Simulated Dataset - Long

\begin{tabular}{|c|c|c|}
\hline id Group & Position & MO Test \\
\hline 1 Control & Full & 14.30 \\
\hline 2 Control & Full & 13.89 \\
\hline 3 Control & Full & 13.81 \\
\hline 4 Control & Full & 15.59 \\
\hline 5 Control & Part & 11.27 \\
\hline 6 Control & Part & 13.22 \\
\hline 7 Control & Part & 14.37 \\
\hline 8 Control & Part & 14.29 \\
\hline 9 Control & Part & 13.49 \\
\hline 10 Control & Part & 14.68 \\
\hline 11 Control & Seasonal & 15.07 \\
\hline 12 Control & Seasonal & 13.64 \\
\hline 13 Control & Seasonal & 14.92 \\
\hline 14 Control & Seasonal & 13.56 \\
\hline 15 Control & Full & 13.91 \\
\hline 16 Treatmen & Full & 14.50 \\
\hline 17 Treatmer & Full & 15.14 \\
\hline 18 Treatmen & Full & 13.72 \\
\hline 19 Treatmer & Full & 13.48 \\
\hline 20 Treatmer & Full & 13.55 \\
\hline 21 Treatmes & Part & 15.92 \\
\hline 22 Trea & Part & 12.83 \\
\hline 23 Treatm & Part & 14.23 \\
\hline 24 Treatmen & Part & 15.04 \\
\hline 25 Treatmen & Seasonal & 14.47 \\
\hline 26 Treatmen & Seasonal & 13.62 \\
\hline 27 Treatmes & Seasonal & 12.42 \\
\hline 28 Treatme & Seasonal & 13.39 \\
\hline 29 Tres & Seasonal & 12.75 \\
\hline 30 Treatmen & Seasonal & 14.95 \\
\hline 1 Control & Full & 25.83 \\
\hline 2 Control & Full & 23.69 \\
\hline 3 Control & Full & 22.97 \\
\hline 4 Control & Full & 25.42 \\
\hline 5 Control & Part & 22.84 \\
\hline 6 Control & Part & 23.06 \\
\hline 7 Control & Part & 23.04 \\
\hline 8 Control & Part & 23.62 \\
\hline 9 Control & Part & 24.41 \\
\hline 10 Control & Part & 24.48 \\
\hline 11 Control & Seasonal & 25.55 \\
\hline 12 Control & Seasonal & 24.15 \\
\hline 13 Control & Seasonal & 24.11 \\
\hline 14 Control & Seasonal & 23.99 \\
\hline 15 Control & Full & 22.85 \\
\hline
\end{tabular}

\begin{tabular}{ll}
\hline id Group Position & MO Test \\
\hline 16 Treatment Full & 26.23 \\
17 Treatment Full & 27.08 \\
18 Treatment Full & 26.92 \\
19 Treatment Full & 25.76 \\
20 Treatment Full & 26.11 \\
21 Treatment Part & 26.69 \\
22 Treatment Part & 26.17 \\
23 Treatment Part & 27.18 \\
24 Treatment Part & 26.21 \\
25 Treatment Seasonal & 24.97 \\
26 Treatment Seasonal & 24.63 \\
27 Treatment Seasonal & 24.07 \\
28 Treatment Seasonal & 26.40 \\
29 Treatment Seasonal & 24.56 \\
30 Treatment Seasonal & 27.01 \\
1 Control Full & 35.12 \\
2 Control Full & 34.39 \\
3 Control Full & 34.59 \\
4 Control Full & 33.96 \\
5 Control Part & 31.97 \\
6 Control Part & 33.55 \\
7 Control Part & 32.82 \\
8 Control Part & 33.48 \\
9 Control Part & 33.84 \\
10 Control Part & 34.24 \\
11 Control Seasonal & 36.26 \\
12 Control Seasonal & 33.07 \\
13 Control Seasonal & 34.12 \\
14 Control Seasonal & 34.24 \\
15 Control Full & 34.35 \\
16 Treatment Full & 35.40 \\
17 Treatment Full & 37.52 \\
18 Treatment Full & 35.33 \\
19 Treatment Full & 34.32 \\
20 Treatment Full & 35.19 \\
21 Treatment Part & 35.96 \\
22 Treatment Treatment Part & 34.34 \\
23 Treatment Part & 36.75 \\
24 Treatment Part & 35.83 \\
25 Treatment Seasonal & 34.59 \\
\hline
\end{tabular}

Note. $\mathrm{MO}=$ measurement occasion $(1=$ Pre; 2 = Post; 3 = FollowUp $)$. 
Nimon et al.

Table 3. Transformation Formulae

\begin{tabular}{|c|c|c|}
\hline Transformation & Formula & Reference \\
\hline$t \rightarrow F$ & $t^{2}$ & \multirow{2}{*}{ Thompson (2006) } \\
\hline$F \rightarrow t$ & $\sqrt{F}$ & \\
\hline$M R^{2} \rightarrow r$ & $\sqrt{M R^{2}}$ & \multirow{4}{*}{ Thompson (2006) } \\
\hline$\eta^{2} \rightarrow r$ & $\sqrt{\eta^{2}}$ & \\
\hline$r \rightarrow R^{2}$ & $r^{2}$ & \\
\hline$r \rightarrow \eta^{2}$ & & \\
\hline$d \rightarrow r$ & $d / \sqrt{d^{2}+\left(N^{2}-2 N\right) /\left(n_{1} n_{2}\right)}$ & \multirow{2}{*}{ McGrath and Meyer (2006) } \\
\hline$r \rightarrow d$ & $\sqrt{-r^{2}\left(N^{2}-2 N\right) /\left(n_{1} n_{2}\right)\left(r^{2}-1\right)}$ & \\
\hline$t_{c} \rightarrow d_{\mathrm{c}}$ & $t_{c} \sqrt{2(1-r) / n}$ & Dunlap et al. (1996) \\
\hline$t \rightarrow d$ & $t / \sqrt{n}$ & Cohen (1988) \\
\hline$\chi^{2} \rightarrow F$ & $\chi^{2} /[($ rows -1$) *($ columns -1$)]$ & \multirow{2}{*}{ Knapp (1978) } \\
\hline$F \rightarrow \chi^{2}$ & $F *($ rows -1$) *($ columns -1$)$ & \\
\hline Cramer's $V \rightarrow M R^{2}$ & Cramer's $V^{2}$ & \multirow{2}{*}{ Cohen (1988) } \\
\hline$M R^{2} \rightarrow$ Cramer's $V^{\prime}$ & $\sqrt{M R^{2}}$ & \\
\hline
\end{tabular}

$2=$ posttest) and individual error (cf. Fox \& Weisberg, 2011). For paired-samples $t$-test, the pretest and posttest scores, respectively, served as the independent and dependent variables. The paired-samples $t$ test models tested the hypothesis that there was a statistically and practically significant mean difference between posttest and pretest scores. Test statistics, $p$ values, effect sizes, and differences between measurement occasion means were compared among the results of the three analyses.

Single-Sample $t$-test. A MLR and a single-sample $t$-test were run testing the hypotheses that pretest scores are statistically and practically different from 0 . For MLR, pretest scores served as the dependent variable, which was modeled only by the intercept. The single-sample $t$-test models tested the hypothesis that the pretest scores were statistically and practically significantly different from 0 . Test statistics, $p$ values, effect sizes, and means were compared between the results of the two analyses.

$\chi^{2}$. Two sets of analyses were run using both chi-square test of independence and MLR. In the first set of analyses, position and group, respectively, served as the dependent and independent variables. The first set of analyses tested the hypothesis that group had a statistically and practically significant effect on position. In the second set of analyses, group and position, respectively, served as the dependent and independent variables. The second set of analyses tested the hypothesis that position had a statistically and practically significant effect on group. In both sets of analyses, the numeric representation of the dependent variable was used for MLR. Test statistics, $p$ values, and effect sizes were compared between the results of the two analyses.

\section{Results}

Appendix $\mathrm{B}$ contains the $\mathrm{R}$ output that resulted from running the syntax in Appendix $\mathrm{A}$. The following sections reference relevant line numbers in Appendix B when presenting the results for each of the analyses demonstrated. Table 3 provides a consolidation of the formulae used to transform statistics and effect sizes.

ANCOVA. Table 4 and Appendix B (lines 95 - 223) present the results of the ANCOVA analyses. The $p$ values for the two analyses (i.e., ANCOVA, MLR) were the same (i.e., 3.22454e-07; see Appendix $\mathrm{B}$, lines $137-141$ ). For ANCOVA, the test statistic produced is an $F$ statistic, whereas a $t$ statistic is produced for the group $b$ weight. As $t^{2}$ is equal to $F$ (Thompson, 2006), the $t$ statistic of 6.723161 is equivalent to the $F$ statistic of 45.20089 (see Appendix B, lines $143-159$ ).

Partial $\eta^{2}$ is the typical effect size reported for ANCOVA, where the variance associated with the covariate (pretest in this case) is excluded from the denominator and only variance associated with the grouping variable (group in this case) is included in the numerator (cf. Thompson, 2006). When using MLR, the partial $\eta^{2}$ can be produced by using commonality analysis coefficients (Zientek, Nimon, \& 
Univariate General Linear Model

Brown, 2016), which can be produced in $\mathrm{R}$ using the calc.yhat function (Nimon, Oswald, \& Roberts, 2013). In the two analyses, the effect sizes produced were identical (i.e., .6260434; see Appendix B, lines $161-186)$.

In ANCOVA, the group means typically reported are means that have been adjusted for their covariate rather Table 4. ANCOVA Results

\begin{tabular}{lrllr}
\multicolumn{2}{c}{ ANCOVA } & & \multicolumn{2}{c}{ MLR } \\
\cline { 1 - 2 } \cline { 5 - 6 } Statistic & Value & & Statistic & Value \\
\hline$p$ & 4.01 & & $p$ & $<.01$ \\
$F$ & .63 & & $\eta_{p}^{2}$ & 6.72 \\
$\eta_{p}^{2}$ & 4.00 & & Adjusted $M_{\text {Ccontrol }}$ & 4.00 \\
Adjusted $M_{\text {Control }}$ & 6.00 & & Adjusted $M_{\text {Treatment }}$ & 6.00 \\
Adjusted $M_{\text {Treatment }}$ & 6.00 & &
\end{tabular}

than the observed means (Nimon \& Henson, 2015; Tracz, Nelson, Newman, \& Beltran, 2005), although in this case there was no difference between observed and adjusted means because the covariate was mean centered. In both analyses, the "adjusted" posttest means were, respectively, 4 and 6 for the control and treatment group. While R provides a function that yields adjusted means via the effect function (Fox, 2003), adjusted means when using MLR require that the intercept and regression weights be used (see Appendix B, lines 188 -222). In summary, group had a statistically and practically significant effect on posttest scores after controlling for pretest scores $\left(t=6.72, F[1,27]=45.2, p<.01 ; \eta_{p}^{2}=.63\right.$; Adjusted $M_{\text {Control }}=4.0$, Adjusted $M_{\text {Treatment }}=6.0$ ).

ANOVA. Table 5 and Appendix B (lines 224 - 314) present the results of the ANOVA and MLR. For the two analyses, results yielded the same values for the test statistic $(F=.33$; see Appendix B, lines $265-270), p$ value $(p=.72$; see Appendix B, lines $259-263)$, and effect size $\left(\eta^{2}=M R^{2}\right.$ $=.02$; see Appendix B, lines $272-276$ ). While the effect size values are identical, the effect size reported for an ANOVA analysis is $\eta^{2}$ and the effect size reported for the MLR analysis is $M R^{2}$. As both effect sizes are varianceexplained statistics, they indicate how much variance in

\begin{tabular}{|c|c|c|c|}
\hline \multicolumn{2}{|c|}{ ANOVA } & \multicolumn{2}{|c|}{ MLR } \\
\hline Statistic & Value & Statistic & Value \\
\hline$p$ & .72 & $p$ & .72 \\
\hline$F$ & .33 & $F$ & .33 \\
\hline$\eta^{2}$ & .02 & $M R^{2}$ & .02 \\
\hline$M_{\text {Full }}$ & 5.29 & $M_{\text {Full }}$ & 5.29 \\
\hline$M_{\text {Part }}$ & 4.77 & $M_{\text {Part }}$ & 4.77 \\
\hline$M_{\text {Seasonal }}$ & 4.94 & $M_{\text {Seasonal }}$ & 4.94 \\
\hline
\end{tabular}
posttest scores was accounted for by group membership. Group means for each analysis were identical with $M_{\text {Full }}=5.29, M_{\text {Part }}=4.77$, and $M_{\text {Seasonal }}=4.94$. Because ANOVA does not provide group means, the values were obtained by calculating descriptive statistics (see Appendix B, lines $278-284$ ). For MLR analyses, the group mean values were obtained by using the intercept and regression coefficients (see Appendix B, lines $285-314)$. In summary, there were no statistically or practically significant mean differences in posttest scores by position $\left(F[2,27]=.33, p=.72 ; \eta^{2}=M R^{2}=.02 ; M_{\text {Full }}=5.29, M_{\text {Part }}=\right.$ 4.77, $\left.M_{\text {Seasonal }}=4.94\right)$.

$\boldsymbol{r}$. Table 6 and Appendix B (lines 316 - 405) present the results of the $r$ and MLR analyses. The $p$ values for the two analyses were the same (i.e., 0.02192; see Appendix B, lines $356-360)$. For $r$, the test statistic produced is a $t$ statistic, whereas an $F$ statistic is produced for the MLR. As $t^{2}$ is equal to $F$ (Thompson, 2006), the $t$ statistic of 2.426894 is equivalent to the $F$ statistic of 5.889816 (see Appendix B,

\begin{tabular}{|lllll|}
\hline \multicolumn{2}{|c|}{ Table 6. $r$ Results } & \multicolumn{2}{c|}{} \\
\cline { 1 - 2 } Statistic & Value & & Statistic & Value \\
\hline$p$ & .02 & & $p$ & .02 \\
$t$ & 2.43 & & $F$ & 5.89 \\
$r$ & .42 & & $M R^{2}$ & .17 \\
\hline
\end{tabular}
lines $362-384$ ). The effect size reported for $r$ is the correlation coefficient $r$, whereas $M R^{2}$ is reported for the MLR. As with the test statistic, the $r^{2}$ is equal to $R^{2}$ (Thompson, 2006). As such, the $r$ of .416885 is equivalent to the $M R^{2}$ of .1737931 (see Appendix B, lines $386-405$ ). In summary, there was a statistically and practically significant relationship between pretest and posttest scores $(t=2.43, F[1,28]$ $\left.=5.89, p=.02 ; r=.42, M R^{2}=.17\right)$.

RM ANOVA. Table 7 and Appendix B (lines 407 - 490) present the results of the RM ANOVA and MLR analysis. The $p$ values $(p=3.799596 \mathrm{e}-05$; see Appendix $\mathrm{B}$, lines $436-441)$, test statistics $(F=$ 12.19; see Appendix B, lines 443 - 447), and effect sizes $\left(\eta_{p}^{2}=.30\right.$; see Appendix B, lines $\left.449-453\right)$ were all identical between the two analyses. In both RM ANOVA and MLR, the partial $\eta^{2}$ is calculated by dividing the amount of variance associated with measurement occasion by the sum of the amount of variance associated with measurement occasion and error (cf. Nimon \& Williams, 2009). Group means 
Nimon et al.

for each analysis were identical with $M_{\text {Pre }}=4.00, M_{\text {Post }}=$ 5.00 , and $M_{\text {FollowUp }}=4.75$. While RM ANOVA does not provide means for each measurement occasion (e.g., pretest, posttest, follow-up), the mean values can be obtained be calculating descriptive statistics (see Appendix $\mathrm{B}$, lines 455 - 460). For the MLR analyses, measurement occasion mean values can be obtained by using the intercept and regression coefficients from a model that regresses the dependent variable on the measurement occasion (see Appendix B, lines 461 - 490). In summary, there were statistically and practically significant mean

\begin{tabular}{|c|c|c|c|}
\hline \multicolumn{4}{|c|}{ Table 7. RM ANOVA Results } \\
\hline \multicolumn{2}{|c|}{ RM ANOVA } & \multicolumn{2}{|c|}{ MLR } \\
\hline Statistic & Value & Statistic & Value \\
\hline$p$ & $<.01$ & $p$ & $<.01$ \\
\hline$F$ & 12.19 & $F$ & 12.19 \\
\hline$\eta_{p}^{2}$ & .30 & $\eta_{p}^{2}$ & .30 \\
\hline$M_{\text {Pre }}$ & 4.00 & $M_{\text {Pre }}$ & 4.00 \\
\hline$M_{\text {Post }}$ & 5.00 & $M_{\text {Post }}$ & 5.00 \\
\hline$M_{\text {Follow Up }}$ & 4.75 & $M_{\text {FollowUp }}$ & 4.75 \\
\hline
\end{tabular}
differences among pretest, posttest, and follow-up scores $\left(F[2,58]=12.19, p<.01 ; \eta_{p}^{2}=.30 ; M_{P r e}=4.0\right.$, $\left.M_{\text {Post }}=5.0, M_{\text {FollowUp }}=4.75\right)$.

Independent Samples $t$-test. Table 8 and Appendix B (lines 492 - 703) present the results of the independent samples $t$-test analyses. The $p$ values for all four analyses (i.e., $t$-test, MLR, ANOVA, and $r$ ) were the same (i.e., 7.537174e-06; see Appendix $\mathrm{B}$, lines 558 - 566). The $t$-test and Table 8. Independent Samples $t$-test Results

\begin{tabular}{|c|c|c|c|c|c|c|c|}
\hline \multicolumn{2}{|c|}{$t$-test } & \multicolumn{2}{|c|}{ MLR } & \multicolumn{2}{|c|}{ ANOVA } & \multicolumn{2}{|l|}{$r$} \\
\hline Statistic & Value & Statistic & Value & Statistic & Value & Statistic & Value \\
\hline$p$ & $<.01$ & $p$ & $<.01$ & $p$ & $<.01$ & $p$ & $<.01$ \\
\hline$t$ & -5.48 & $F$ & 30.00 & $F$ & 30.00 & $t$ & 5.48 \\
\hline$d$ & 2.00 & $M R^{2}$ & .52 & $\eta^{2}$ & .52 & $r$ & .72 \\
\hline$M_{\text {Control }}$ & 4.00 & $M_{\text {Control }}$ & 4.00 & $M_{\text {Control }}$ & 4.00 & $M_{\text {Control }}$ & 4.00 \\
\hline$M_{\text {Treatment }}$ & 6.00 & $M_{\text {Treatment }}$ & 6.00 & $M_{\text {Treatment }}$ & 6.00 & $M_{\text {Treatment }}$ & 6.00 \\
\hline
\end{tabular}
$r$ provide a $t$-test statistic, whereas MLR and ANOVA provide an $F$ statistic. As $t^{2}$ is equal to $F$ (Thompson, 2006), the $t$ statistics of -5.477226 and 5.47726 are equivalent to the $F$ statistic of 30.0 (see Appendix B, lines 568-605). Note that the test statistic for the $t$-test is negative while positive for $r$ (see Appendix B, lines $571 \& 579$ ). This is because the mean for the first group (Control) was less than the mean for the second group (Treatment), and there was a positive relationship between posttest scores and the numeric representation of group since group was coded as 1 and treatment was coded as 2 .

The typical effect size reported for an independent samples $t$-test is Cohen's $d$, where the mean difference is divided by the pooled standard deviation (Cohen, 1988). The MLR, ANOVA, and $r$, respectively, yielded $M R^{2}, \eta^{2}$, and $r$. Whereas the $R^{2}$ and the $\eta^{2}$ are already in a comparable dimension and equal at .517241, the Cohen's $d$ of 2 was converted to $r$ (Lakens, 2013; McGrath \& Meyer, 2006), resulting in .719195 , which is equivalent to the $M R^{2}$ of .517241 for reasons previously stated (see Appendix B, lines $607-655$ ). Group means for each analysis are also identical where $M_{\text {Control }}=4.0$ and $M_{\text {Treatment }}=6.0$. As ANOVA and $r$ do not provide group means or information to compute group means, descriptive statistics were calculated (see Appendix B, lines 696-703). For MLR analyses, group mean values were obtained by using the intercept and regression coefficients (see Appendix B, lines 671 - 695). In summary, there was a statistically and practically significant mean difference in posttest scores by group $\left(t[28]= \pm 5.48, F[1,28]=30.00, p<.01 ; d=2.00, r=.72, M R^{2}=\eta^{2}=.52 ; M_{\text {Control }}=4.00\right.$, $M_{\text {Treatment }}=6.00$ ).

Paired-Samples $\boldsymbol{t}$-test. Table 9 and Appendix B (lines $705-813$ ) present the results of the paired-samples $t$-test analyses. The $p$ values for all three analyses (i.e., $t$-test, MLR, and RM ANOVA) were the same (i.e., .000327; see Appendix B, lines 757 - 764). The $t$-test and the MLR provide a $t$ statistic whereas RM ANOVA provides an $F$ statistic. As $t^{2}$ is equal

\begin{tabular}{|c|c|c|c|c|c|}
\hline \multicolumn{2}{|c|}{$t$-test } & \multicolumn{2}{|c|}{ MLR } & \multicolumn{2}{|c|}{ RM ANOVA } \\
\hline Statistic & Value & Statistic & Value & Statistic & Value \\
\hline$p$ & $<.01$ & $p$ & $<.01$ & $p$ & $<.01$ \\
\hline$t$ & 4.07 & $t$ & 4.07 & $F$ & 16.60 \\
\hline$d$ & .80 & $d$ & .80 & $d$ & .80 \\
\hline$M_{\text {Post-Pre }}$ & 1.00 & $M_{\text {Post-Pre }}$ & 1.00 & $M_{\text {Post-Pre }}$ & 1.00 \\
\hline
\end{tabular}
to $F$ (Thompson, 2006), the $t$ statistic of 4.074684 is equivalent to the $F$ statistic of 16.60305 (see Appendix B, lines 766 - 794). The effect size for each analysis was also identical (i.e., Cohen's $d=$ .803388; see Appendix B, lines 796 - 804). Cohen's $d$ was calculated using the formula for matched groups (Dunlap, Cortina, Vaslow, \& Burke, 1996). The mean difference between posttest and pretest 
scores was identical for each analysis (i.e., $M_{\text {Post-Pre }}=1$; see Appendix B, lines $806-813$ ). Because ANOVA does not provide group means, descriptive statistics were calculated on posttest minus pretest scores. For MLR, the intercept provided the mean difference between posttest and pretest scores. In summary, there was a statistically and practically significant mean difference between posttest and pretest scores $\left(t[29]=4.07, F[1,29]=16.60, p<.01 ; d=.80 ; M_{\text {Post-Pre }}=1.00\right)$.

Single-Sample $t$-test. Table 10 and Appendix B (lines 815 - 876) present the results of the single-sample $t$-test analyses. The $p$ values for both analyses (i.e., $t$-test, MLR) were the same (i.e., 8.45791e-20; see Appendix B, lines $852-856)$. The $t$-test and the MLR produced identical $t$ statistics (i.e., 22.2967; see Appendix B, lines 858-863). The effect size for each analysis was also identical (i.e., Cohen's $d=4.070802$; see Appendix B, lines $865-869$ ). For the single-sample $t$-test, Cohen's $d$ was calculated by

\begin{tabular}{|c|c|c|c|}
\hline $\begin{array}{r}\text { Table } 1 \\
t-\mathrm{t}\end{array}$ & Singl & $\frac{\text { mple } t \text {-te }}{\mathrm{Ml}}$ & Resu \\
\hline Statistic & Value & Statistic & Value \\
\hline$p$ & $<.01$ & $p$ & $<.01$ \\
\hline$t$ & 22.30 & $t$ & 22.30 \\
\hline$d$ & 4.07 & $d$ & 4.07 \\
\hline$M_{\text {Pre }}$ & 4.00 & $M_{\text {Pre }}$ & 4.00 \\
\hline
\end{tabular}
dividing the $M$ by the $S D$ of pretest scores. For MLR, Cohen's $d$ was calculated using the $t$ statistic and formula for one-sample $t$-test (Cohen, 1988, p. 72). The mean pretest score was identical for each analysis (i.e., $M_{\mathrm{Pre}}=4.00$; see Appendix B, lines $871-876$ ). For MLR, the intercept provided the mean pretest score. In summary, the mean pretest score was statistically and practically significant different from $0\left(t[29]=22.30, p<.01 ; d=4.07 ; M_{\mathrm{Pre}}=4.00\right)$.

$\chi^{2}$ Table 11 and Appendix B (lines $878-$ 1072) present the results of the $\chi^{2}$ and MLR analyses. To demonstrate that MLR does not subsume $\chi^{2}$ analyses in all cases, we first modeled position by group, which considered a

\begin{tabular}{|c|c|c|c|c|c|c|c|}
\hline \multicolumn{8}{|c|}{ Table 11. $\chi^{2}$ Results } \\
\hline \multicolumn{4}{|c|}{ Position Group } & \multicolumn{4}{|c|}{ Group Position } \\
\hline \multicolumn{2}{|c|}{$\chi^{2}$} & \multicolumn{2}{|c|}{ MLR (Incorrect) } & \multicolumn{2}{|c|}{$\chi^{2}$} & \multicolumn{2}{|c|}{ MLR (Correct) } \\
\hline Statistic & Value & Statistic & Value & Statistic & Value & Statistic & Value \\
\hline$p$ & .67 & $p$ & .67 & $p$ & .67 & $p$ & .69 \\
\hline$\chi^{2}$ & .80 & $F$ & .19 & $\chi^{2}$ & .80 & $F$ & .37 \\
\hline Cramer's $V$ & .16 & $M R^{2}$ & .01 & Cramer's $V$ & .16 & $M R^{2}$ & .03 \\
\hline
\end{tabular}
$3 \times 2$ association. Using MLR to analyze a 3x2 association is not valid for multiple reasons. First, MLR does not accept categorical data as a dependent variable. Second, modeling the numeric representation of a variable with more than two categorical levels (e.g., position) is not appropriate and returned erroneous results, as depicted in Table 11 and Appendix B (lines 897 - 966).

To demonstrate that MLR does subsume $\chi^{2}$ analyses in certain cases, we modeled group by position (Group Position), which considered a $2 \times 3$ association where group was treated as a dichotomous dependent variable (see Appendix B, lines 968 - 1072). The group by position results are provided in the Group Position column of Table 11. The chi-square test returned 2 degrees of freedom $(d f=$ [rows -1 ] [columns -1$]$ ) and the MLR returned $d f_{\text {error }}=27$, where the latter took into consideration the number of predictors $(k=2)$ and sample size $(n=30)$. As well, the two approaches to the $\chi^{2}$ analysis delivered different $p$ values (see Appendix B, lines 1026 - 1030). This difference in $p$ value is attributed to the fact that chi-square probability calculations are not sensitive to sample size (McNeil, 1974). In this example, the probability statistic from the MLR (i.e., .694) can be considered more accurate than from the chisquare (i.e., .670) due to the small sample size of 30 . The chi-square probability value "becomes more exact when larger sample sizes are observed" (McNeil, p. 53).

Similar to the other analyses, different test statistics were returned. The chi-square test yielded a $\chi^{2}$ statistic (i.e., .80), and the MLR yielded an $F$ statistic (i.e., .37). When converted using Knapp's (1978) formula and its derivative (see Table 3), these test statistics are approximately equal (see Appendix B, lines 1032 - 1054). Effect sizes produced by the analyses are also different but equivalent. The chisquare test produced a Cramer's $V$ (i.e., .163), and the MLR produced an $M R^{2}$ (i.e., .027). Once the Cramer's $V$ is squared, the observed effect sizes are identical (Cohen, 1988, see Appendix B, lines 1056 1072). In summary, position did not have a statistically or practically significant effect on group $\left(\chi^{2}[2]=\right.$ $.80, p=.67 ; F[2,27]=.37, p=.694$; Cramer's $\left.V=.16, M R^{2}=.03\right)$. 
Nimon et al.

\section{Discussion}

The content presented in this article affords graduate students and emerging scholars a cogent illustration of how MLR subsumes univariate analyses in the GLM. In addition to the illustration, the present paper extends current literature by demonstrating how (a) independent samples $t$-test is subsumed by both ANOVA and Pearson's $r$, (b) RM ANOVA is subsumed by MLR and subsequently subsumes paired-samples $t$-test, (c) MLR subsumes single-samples $t$-test, and (d) MLR subsumes chi-square only in special cases. Researchers may utilize the content herein as a reference guide since it provides (a) a more rigorous visual representation of the univariate GLM, (b) an explanation of the test statistics and effect sizes yielded by comparable statistical analyses, (c) a complete table of transformation formulae with pertinent references, (d) example write-ups that accompany each set of analyses, and (e) replicable syntax that may be copied, modified, and applied to other research studies.

Novice readers of academic literature describing the GLM may interpret the arguments presented as doctrine without fully understanding and exploring the underlying concepts. This article attempts to guide the novice reader through the hierarchical nature of the univariate GLM by demonstrating the analyses which may be replicated and compared using the syntax and output provided. If readers undergo the replication process afforded, they should recognize that MLR does, in fact, subsume the univariate parametric analyses within the GLM. Through this exploration, replication, and independent study, readers will likely better understand the arguments and concepts that connect the univariate GLM analyses.

The statistical analyses presented in this paper are often described as independent tools that are used for specific purposes. In reality, and due to their inherent incorporation within the GLM, MLR is not unidimensional in its application. We expect that prudent researchers will understand the similarities, differences, and limitations (e.g., chi-square's sensitivity to sample size) of the univariate GLM analyses and will apply the appropriate analysis to best match their research design and data.

The paper indirectly reinforces the concept that statistics do not determine causality. Although MLR is often maligned for not yielding experimental evidence (e.g., Nisbett, 2016), readers should understand that data from an experimental design could be analyzed with MLR and therefore yield experimental evidence. Also, the paper demonstrates why statements such as "correlation never implies causality" are wrong (cf. Huck, 2012). Only aspects of research design determine causality, not the statistics used to analyze the data yielded from the research design.

This paper is not without limitations. First, it considered only the univariate GLM and did not demonstrate how canonical correlation subsumes the multivariate and univariate analyses. Nor did it demonstrate SEM as the most general form of the GLM or consider other univariate analyses including split-plot ANOVA. Second, the paper provided only R syntax to accompany the analyses. Third, the data used to demonstrate the GLM were simulated and designed to meet the statistical assumptions of the analyses. As such, the syntax did not include checks for the statistical assumptions for each analysis. Future research could consider building on the work presented in this paper by addressing the aforementioned limitations.

\section{References}

Bagozzi, R. P., Fornell, C., \& Larcker, D. F. (1981). Canonical correlation analysis as a special case of a structural relations model. Multivariate Behavioral Research, 16, 437-454. doi:10.1207/s15327906mbr1604_2

Cohen, J. (1968). Multiple regression as a general data-analytic system. Psychological Bulletin, 70, 426433. doi:10.1037/h0026714

Cohen, J., (1988). Statistical power and analysis for the behavioral sciences (2nd ed.). Hillsdale, NJ: Erlbaum.

Crawley, M. J. (2013). The R book. West Sussex, England: Wiley.

Dunlap, W. P., Cortina, J. M., Vaslow, J. B., \& Burke, M. J. (1996). Meta-analysis of experiments with matched groups or repeated measures designs. Psychological Methods, 1, 170-177. doi:10.1037/1082-989x.1.2.170

Fox, J. (2003). Effect displays in R for generalised linear models. Journal of Statistical Software, 8, 1-27. doi:10.18637/jss.v008.i15 
Fox, J., \& Weisberg, S. (2011). An $R$ companion to applied regression (2nd ed.). Thousand Oaks, CA: Sage.

Graham, J. M. (2008). The general linear model as structural equation modeling. Journal of Educational and Behavioral Statistics, 33, 485-506. doi: 10.3102/1076998607306151

Henson, R. K., Hull, D. M., \& Williams, C. S. (2010). Methodology in our education research culture: Toward a stronger collective quantitative proficiency. Educational Researcher, 39, 229-240. doi:10.3102/0013189x10365102

Huck, S. W. (2012). Reading statistics and research (6th ed.). Boston, MA: Allyn \& Bacon.

Knapp, T. R. (1978). Canonical correlation analysis: A general parametric significance-testing system. Psychological Bulletin, 85, 410-416. doi:10.1037/0033-2909.85.2.410

Lakens, D. (2013). Calculating and reporting effect sizes to facilitate cumulative science: A practical primer for t-tests and ANOVAs. Frontiers in Psychology, 4, 863. doi:10.3389/fpsyg.2013.00863

McGrath R. E., \& Meyer G. J. (2006). When effect sizes disagree: The case of $\mathrm{r}$ and d. Psychological Methods, 11, 386-401. doi: 10.1037/1082-989X.11.4.386

McNeil, K. (1974). The multiple linear regression approach to "chi square" hypotheses. Journal of Experimental Education, 43, 53-55. doi: 10.1080/00220973.1974.10806319

Nimon, K., \& Henson, R. (2015). Validity of a residualized dependent variable after pretest covariance correction: Still the same variable? Journal of Experimental Education, 18, 405-422. doi:10.1080/00220973.2014.907228

Nimon, K., Oswald, F. L., \& Roberts, J. K. (2013). Yhat: Interpreting regression effects. R Packaged version, 2-0, http://CRAN. R-project.org/package= yhat

Nimon, K., \& Williams, C. (2009). Performance improvement through repeated measures: A primer for educators considering univariate and multivariate design. Research in Higher Education Journal, 2, $117-136$.

Nisbett, R. (2016). The crusade against multiple regression analysis: A conversation with Richard Nisbett. Retrieved from https://www.edge.org/conversation/richard_nisbett-the-crusade-againstmultiple-regression-analysis

Osborne, J. W. (2013). Normality of residuals is a continuous variable, and does seem to influence the trustworthiness of confidence intervals: A response to, and appreciation of, Williams, Grajales, and Kurkiewicz (2013). Practical Assessment, Research \& Evaluation, 18(12), 1-9.

R Development Core Team. (2017). R: A language and environment for statistical computing [Computer software]. Vienna, Austria: R Foundation for Statistical Computing. Retrieved from http://www.Rproject.org/

Thompson, B. (2006). Foundations of behavioral statistics. New York: Guilford.

Tracz, S. M., Nelson, L. L., Newman, I., \& Beltran, A. (2005). The misuse of ANCOVA: The academic and political implications of Type VI errors in studies of achievement and socioeconomic status. Multiple Linear Regression Viewpoints, 31, 16-21.

White, D. (2015). Active learning and threshold concepts in multiple testing that can further develop student critical statistical thinking. Teaching Statistics, 37, 48-53. doi:10.1111/test.12069

Zientek, L. R., Nimon, K., \& Brown, B. (2016). Analyzing data from a pretest-posttest control group design: The importance of statistical assumptions. European Journal of Training and Development, 40, 638-659. doi:10.1108/ejtd-08-2015-0066

Zientek, L. R., \& Thompson, B. (2009). Matrix summaries improve research reports: Secondary analyses using published literature. Educational Researcher, 38, 343-352. doi: 10.3102/0013189X09339056.

Send correspondence to:

Kim Nimon

University of Texas at Tyler

Email: kim.nimon@gmail.com 
Nimon et al.

\section{APPENDIX A}

\section{R Software to Replicate Reported Analyses}

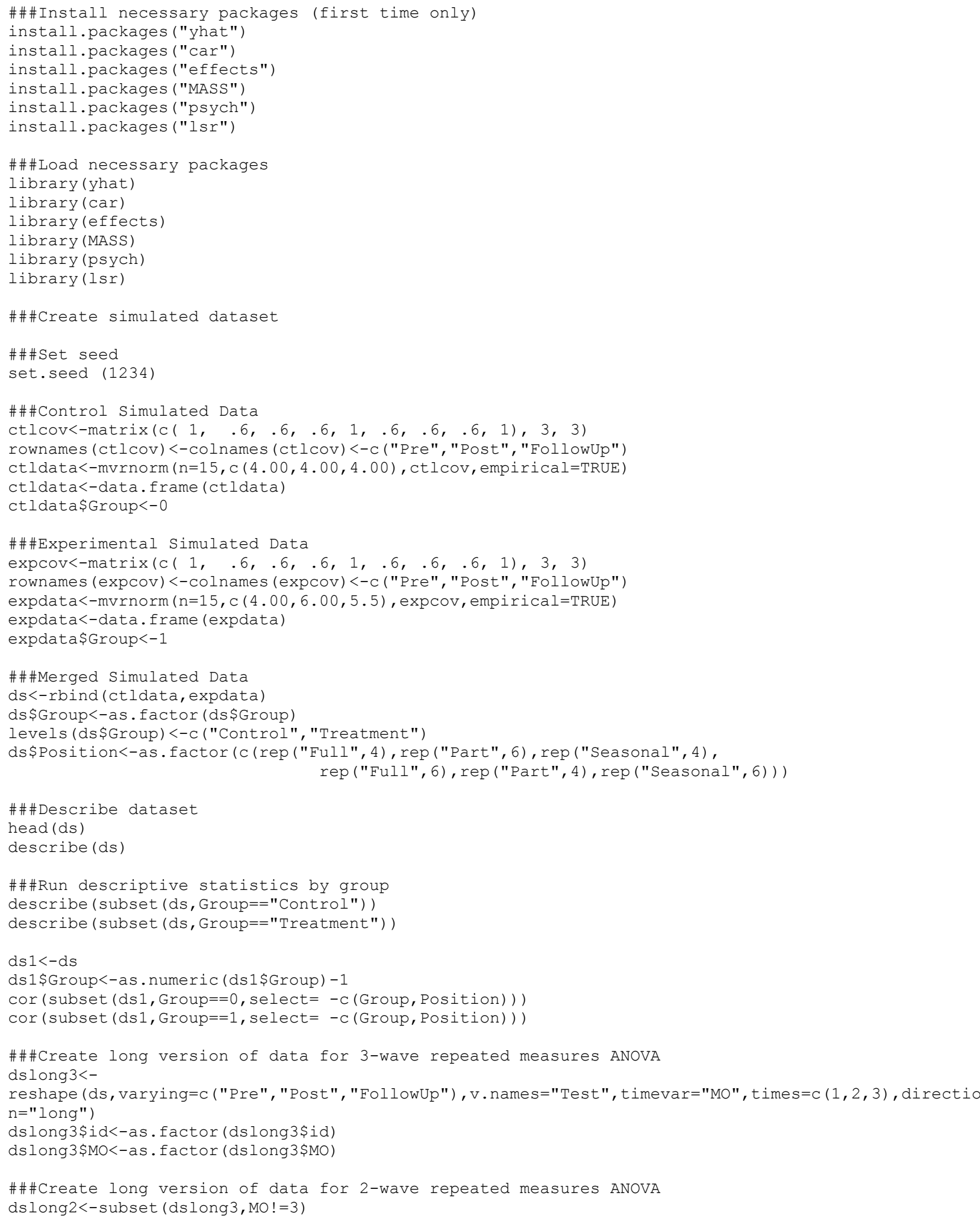




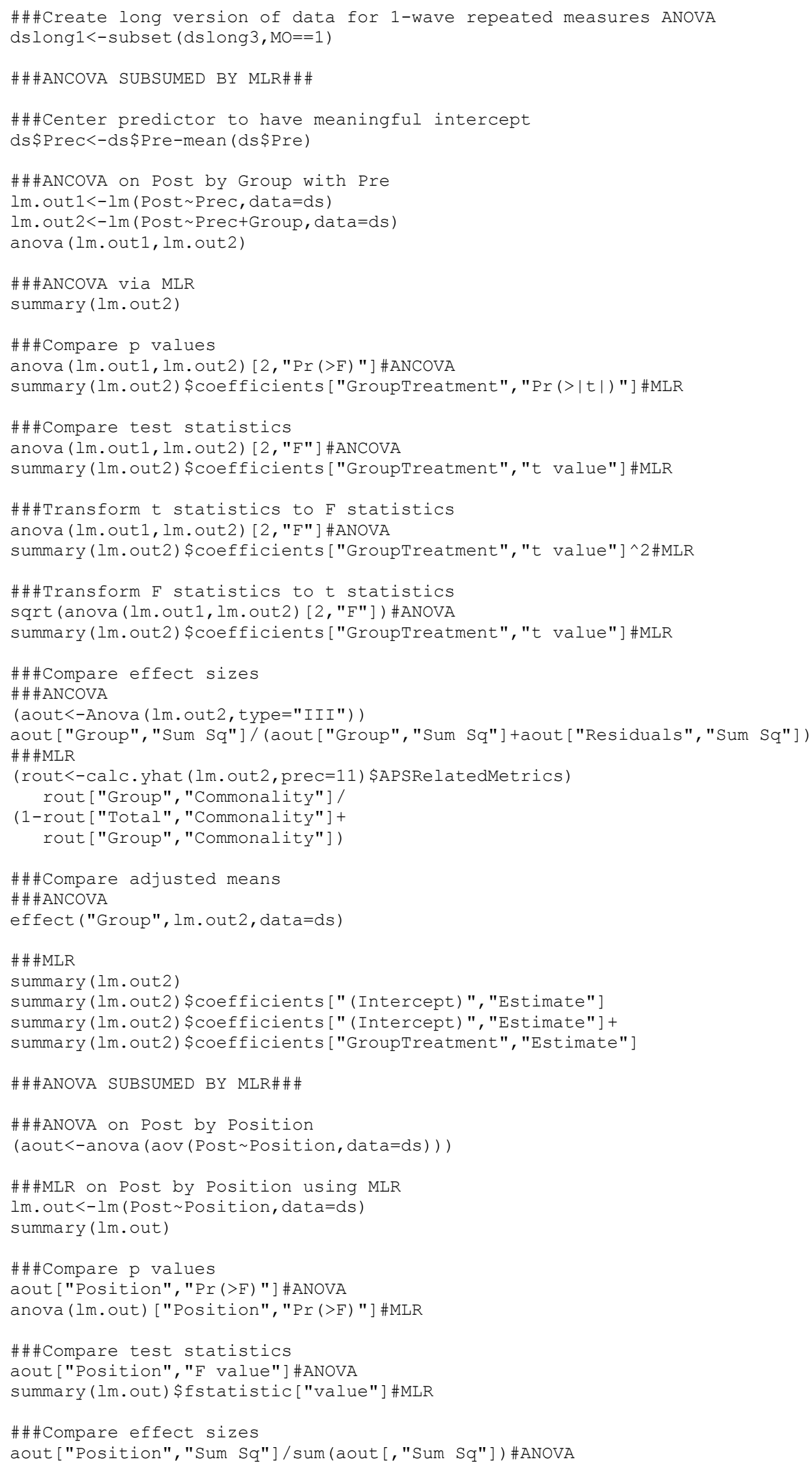


Nimon et al.

summary (lm.out) \$r.squared\#MLR
\#\#\#Compare group means
\#\#\#ANOVA
aggregate (ds\$Post ds\$Position, ds, mean)
\#\#\#MLR
summary (lm.out)
summary(lm.out) \$coefficients [" (Intercept)", "Estimate"]
summary(lm.out) \$coefficients [" (Intercept)","Estimate"]+
summary(lm.out) \$coefficients ["PositionPart","Estimate"]
summary(lm.out) \$coefficients ["(Intercept)","Estimate"]+
summary(lm.out) \$coefficients ["PositionSeasonal","Estimate"]

\#\#\# SUBSUMED BY MLR\#\#\#

\#\#\#correlation between Post and Pre using Pearson's

(cor.out<-cor.test (ds\$Post, ds\$Pre))

\#\#\#correlation between Post and Group using MLR

lm. out $<-\operatorname{lm}$ (Post $\sim$ Pre, data $=$ ds $)$

summary ( $1 \mathrm{~m}$. out)

\#\#\#Compare p values

cor.out\$p.value\#Pearson's r

anova (lm.out) ["Pre", "Pr (>F)"] \#MLR

\#\#\#Compare test statistics

cor.out\$statistic\#Pearsons's r

summary (lm.out) \$fstatistic ["value"] \#MLR

\#\#\#ransform to $\mathrm{F}$

cor.out\$statistic^2\#Pearsons $r$

summary(lm.out) \$fstatistic ["value"] \#MLR

\#\#\#Transform $\mathrm{F}$ to $t$

cor.out\$statistic\#Pearson's r

sqrt (summary(lm.out) \$fstatistic ["value"]) \#MLR

\#\#\#Compare effect sizes

cor.out\$estimate\#Pearson's r

summary (lm.out) \$r.squared\#MLR

\#\#\#ransform $r$ to $\mathrm{R} 2$

cor.out\$estimate^2\#Pearson's r

summary (lm.out) \$r.squared\#MLR

\#\#\#ransform R2 to $r$

cor.out\$estimate\#Pearson's r

sqrt (summary (lm. out) \$r.squared) \#MLR

\#\#\#REPEATED MEASURES ANOVA SUBSUMED BY MLR\#\#\#

\#\#\#Repeated Measures ANOVA on Pre, Post, and Followup

aoutrm<-aov (Test MO+Error (id), data=dslong3)

summary (aoutrm)

\#\#\#LR on Pre, Post, and Followup

(aoutmlr<-anova ( $\mathrm{lm}($ Test $\sim \mathrm{MO}+\mathrm{id}$, data=dslong3)) )

\#\#\#Compare p values

( (a<-unlist (summary (aoutrm) [ ["Error: Within"] ])) ["Pr (>F) 1"]) \#ANOVA

aoutmlr ["MO", "Pr $(>\mathrm{F})$ "]

\#\#\#Compare test statistic

a [ ["F value1"] ]\#RM ANOVA

aoutmlr["MO","F value"] \#MLR

\#\#\#Compare effect sizes

a [["Sum Sq1"]]/(a [["Sum Sq1"]]+a[["Sum Sq2"]]) \#RM ANOVA

aoutmlr["MO", "Sum Sq"]/(aoutmlr["MO","Sum Sq"]+aoutmlr["Residuals", "Sum Sq"]) \#MLR 
\#\#\#Compare measurement occasion means

aggregate (Test $\sim \mathrm{MO}$, dslong3, mean) \#RM ANOVA

lm. out $<-\operatorname{lm}($ Test $\sim \mathrm{MO}$, data=dslong 3$)$

summary (lm.out)

summary (lm.out) \$coefficients [" (Intercept)","Estimate"]

summary (lm.out) \$coefficients [" (Intercept)","Estimate"]+

summary (lm.out) \$coefficients ["MO2", "Estimate"]

summary (lm.out) \$coefficients [" (Intercept)","Estimate"] +

summary (lm.out) \$coefficients ["MO3", "Estimate"]

\#\#\#INDEPENDENT T TEST SUBSUMED BY MLR, ANOVA, AND r\#\#\#

\#\#\#-test on Post by Group

( $t$. out<-t. test (Post Group, data=ds, paired=FALSE, var. equal=TRUE) )

\#\#\#MLR on Post by Group

lm. out $<-\operatorname{lm}$ (Post $\sim$ Group, data $=$ ds $)$

summary ( $1 \mathrm{~m}$. out)

\#\#\#ANOVA on Post by Group

(aout<-anova (aov (Post Group, data=ds)) )

\#\#\#correlation between Post and Group using Pearson's $r$

(cor.out<-cor.test (ds\$Post, as.numeric (ds\$Group)))

\#\#\#Compare p values

t.out\$p.value\#t-test

anova (lm.out) ["Group", "Pr (>F)"] \#MLR

aout ["Group", "Pr $(>\mathrm{F})$ "] \#ANOVA

cor.out\$p.value\#Pearson's r

\#\#\#Compare test statistic

t.out\$statistic\#t-test

summary (lm.out) \$fstatistic ["value"] \#MLR

aout ["Group", "F value"] \#ANOVA

cor.out\$statistic\#Pearson's r

\#\#\#Transform t to $\mathrm{F}$

t.out\$statistic^2\#t-test

summary (lm.out) \$fstatistic ["value"] \#MLR

aout ["Group", "F value"] \#ANOVA

cor.out\$statistic^2\#Pearson's r

\#\#\#Tansform $\mathrm{F}$ to $t$

abs (t.out\$statistic) \#t-test

sqrt (summary (lm.out) \$fstatistic ["value"]) \#MLR

sqrt (aout ["Group", "F value"]) \#ANOVA

cor.out\$statistic\#Pearson's r

\#\#\#Compare effect sizes

( $d<-$ cohensD (ds\$Post ds $\$$ Group) ) \#t-test

(r2<-summary ( $1 \mathrm{~m}$. out) \$r. squared) \#MLR

(e2<-aout ["Group", "Sum Sq"] / sum (aout [,"Sum Sq"])) \#ANOVA

cor.out\$estimate\#Pearson's r

\#\#\#ransform d, eta-squared, and R2 to $r$

$d / \operatorname{sqrt}(d * * 2+((\operatorname{nrow}(d s) * * 2-2 * \operatorname{nrow}(d s)) /($ table $(d s \$ G r o u p)[1] * t a b l e(d s \$ G r o u p)[2]))) \# t-t e s t$

(tr $1<-\operatorname{sqrt}(r 2))$ \#MLR

(tr2<-sqrt (aout ["Group", "Sum Sq"] / sum (aout [, "Sum Sq"]))) \#ANOVA

(tr3<-cor.out\$estimate) \#Pearson's r

\#\#\#Transform R2, eta-squared, and $r$ to $d$

d\#t-test

$\operatorname{sqrt}((-\operatorname{tr} 1 * \star 2 *(\operatorname{nrow}(\mathrm{ds}) * \star 2-2 * \operatorname{nrow}(\mathrm{ds}))) /(\operatorname{table}(\mathrm{ds} \$ \mathrm{Group})[1]$ *table (ds $\$ \mathrm{Group})[2]$ * (tr $1 * \star 2-1))) \# \mathrm{MLR}$

$\operatorname{sqrt}((-\operatorname{tr} 2 * * 2 *(\operatorname{nrow}(d s) * * 2-2 * \operatorname{nrow}(d s))) /(\operatorname{table}(\mathrm{ds} \$ \operatorname{Group})[1] * \operatorname{table}(\operatorname{ds} \$ \mathrm{Group})[2] *(\operatorname{tr} 2 * * 2-1))) \#$ ANOVA sqrt $((-\operatorname{tr} 3 * * 2 *(\operatorname{nrow}(d s) * * 2-2 * \operatorname{nrow}(d s))) /(\operatorname{table}(\mathrm{ds} \$ \mathrm{Group})[1] * \operatorname{table}(\mathrm{ds} \$ \mathrm{Group})[2] *(\operatorname{tr} 3 * \star 2-$

1))) \#Pearson's r

\#\#\#Transform d and $r$ to $\mathrm{R} 2 /$ eta-squared

$(d / \operatorname{sgrt}(d * * 2+((\operatorname{nrow}(d s) * * 2-2 * \operatorname{nrow}(d s)) /($ table (ds\$Group) [1] *table (ds\$Group) [2] )) ) **2\#t-test r2\#MLR 


\section{Nimon et al.}

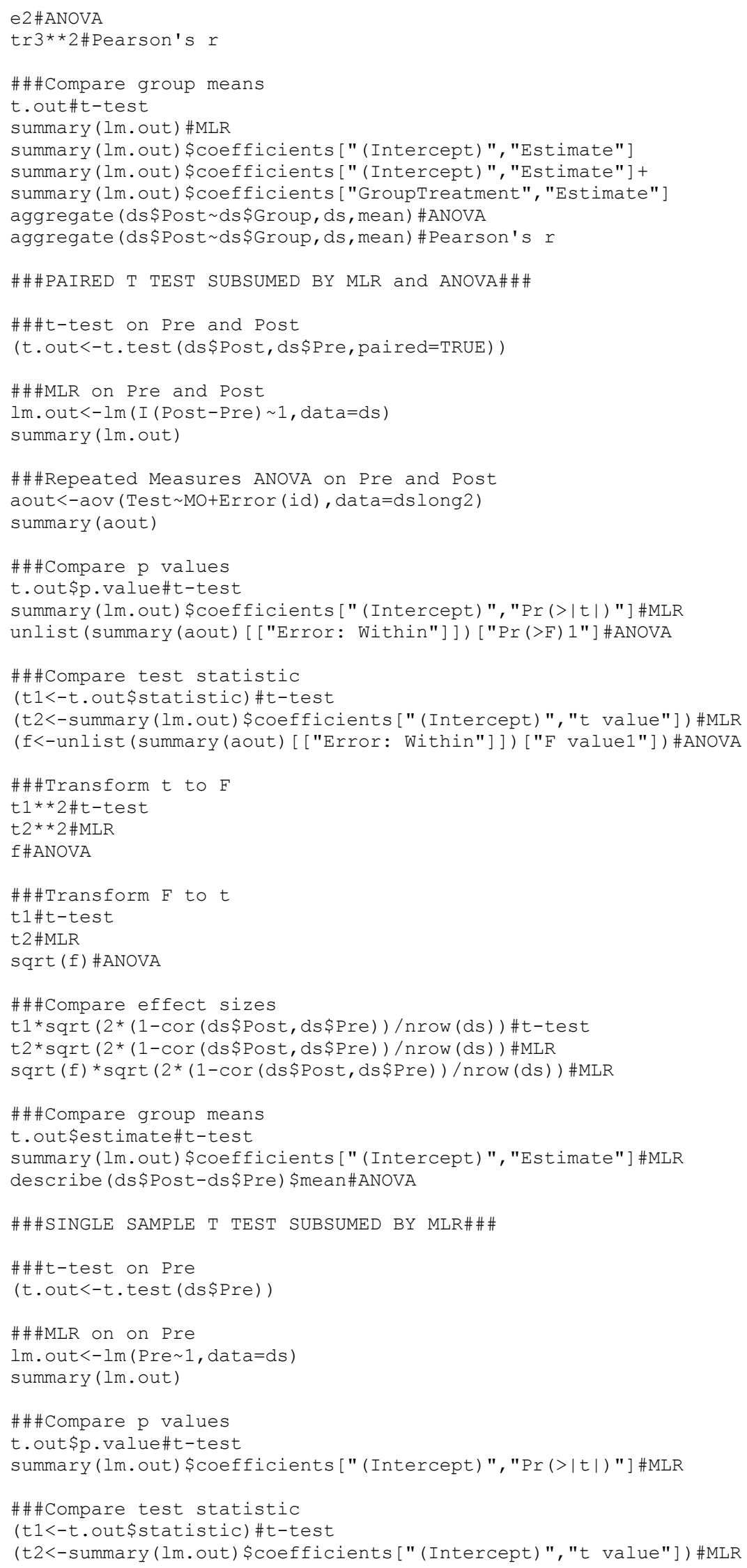




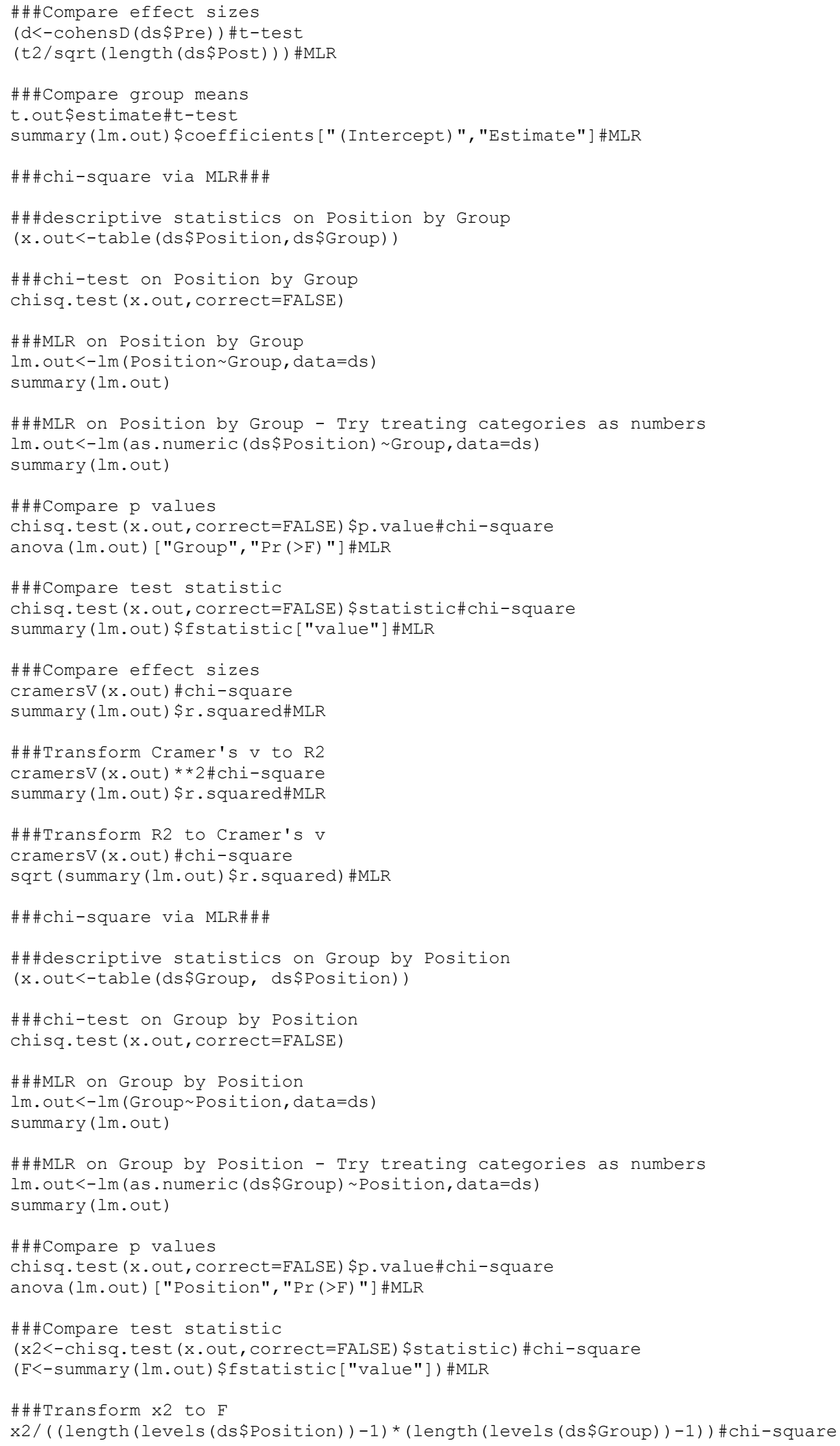


Nimon et al.

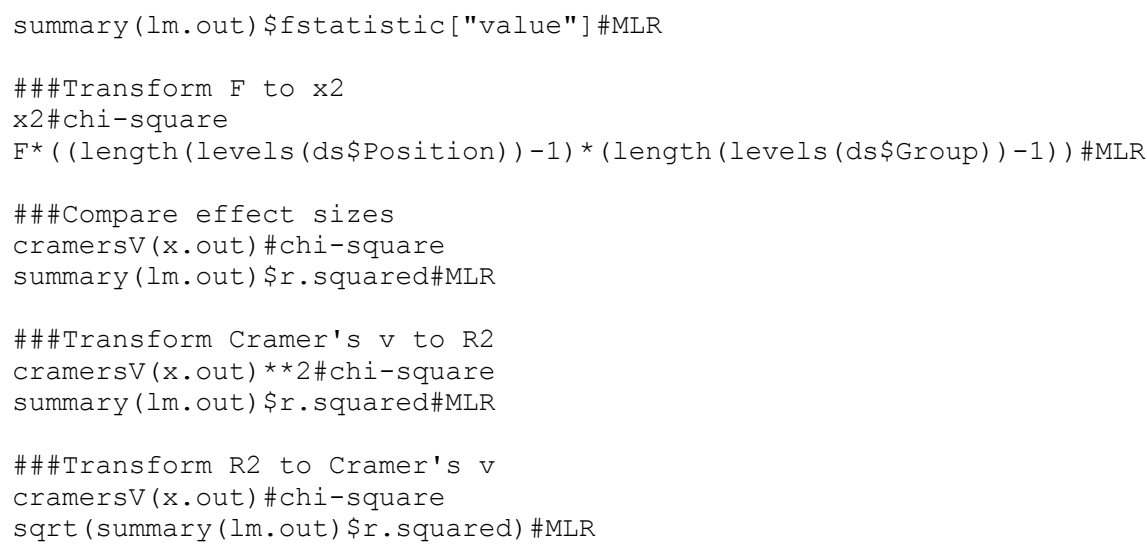


$>$ \#\#\#Load necessary packages

\section{APPENDIX B \\ R Output for Illustrative Examples}

$>$ library (yhat)

$>$ library (car)

$>$ library (effects)

$>$ library (MASS)

$>$ library (lsr)

$>$

$>$ \#\#\#Create simulated dataset

\#\#\#Set seed

set.seed (1234)

$>$ \#\#\#Control Simulated Data

$>$ ctlcov<-matrix $(\mathrm{c}(1, .6, .6, .6,1, .6, .6, .6,1), 3,3)$

$>$ rownames (ctlcov)<-colnames (ctlcov)<-c ("Pre", "Post", "FollowUp")

$>$ ctldata<-mvrnorm ( $\mathrm{n}=15, \mathrm{c}(4.00,4.00,4.00)$, ctlcov, empirical=TRUE)

$>$ ctldata<-data.frame (ctldata)

$>$ ctldata $\$$ Group $<-0$

$>$

$>$ \#\#\#Experimental Simulated Data

$>$ expcov<-matrix $(c(1, .6, .6, .6,1, .6, .6, .6,1), 3,3)$

$>$ rownames (expcov)<-colnames (expcov)<-c ("Pre", "Post", "FollowUp")

$>$ expdata<-mvrnorm ( $\mathrm{n}=15, \mathrm{c}(4.00,6.00,5.5)$, expcov, empirical=TRUE)

$>$ expdata<-data.frame (expdata)

$>$ expdata $\$$ Group $<-1$

$>$

$>$ \#\#\#Merged Simulated Data

$>$ ds<-rbind (ctldata, expdata)

$>$ ds\$Group<-as.factor (ds\$Group)

$>$ levels (ds\$Group) <-c ("Control", "Treatment")

$>$ ds\$Position<-as.factor (c (rep ("Full", 4), rep ("Part", 6), rep ("Seasonal", 4),

$+\operatorname{rep}(" F u l l ", 6), \operatorname{rep}(" P a r t ", 4), \operatorname{rep}(" S e a s o n a l ", 6)))$

$>$ \#\#\#Describe dataset

$>\operatorname{head}(\mathrm{ds})$

Pre Post FollowUp Group Position

14.2968385 .8288095 .122568 Control Full

23.8898973 .6886674 .390797 Control Full

$3 \quad 3.8131392 .9675884 .589542$ Control Full

$45.5877085 .424477 \quad 3.962162$ Control Full

51.2671852 .8399001 .970185 Control Part

63.2178343 .0565023 .548479 Control Part

$>$ describe (ds)

vars $n$ mean sd median trimmed mad min max range skew kurtosis se

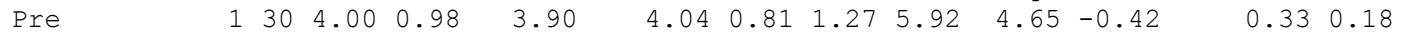

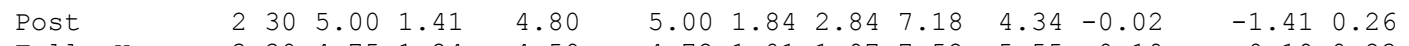

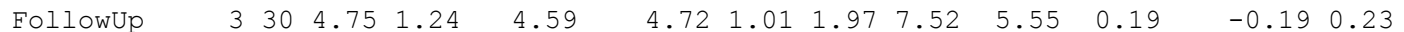

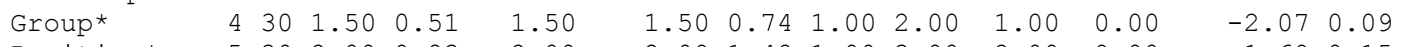

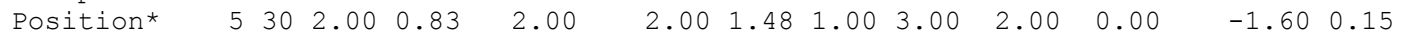

$>$

$>$ \#\#\#Run descriptive statistics by group

$>$ describe (subset (ds, Group=="Control"))

vars $n$ mean sd median trimmed mad min max range skew kurtosis se

$\begin{array}{lllllllllllllll}\text { Pre } & 1 & 15 & 4.00 & 1.0 & 3.91 & 4.09 & 0.63 & 1.27 & 5.59 & 4.32 & -0.98 & 1.33 & 0.26\end{array}$

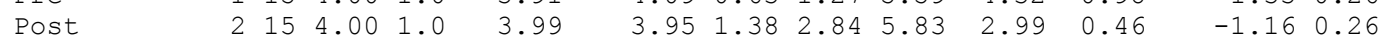

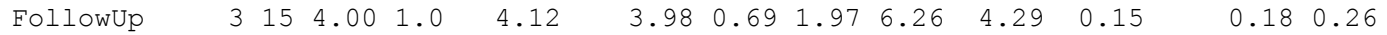

$\begin{array}{llllllllllllllll}\text { Group* } & 4 & 15 & 1.00 & 0.0 & 1.00 & 1.00 & 0.00 & 1.00 & 1.00 & 0.00 & \mathrm{NaN} & \mathrm{NaN} & 0.00\end{array}$

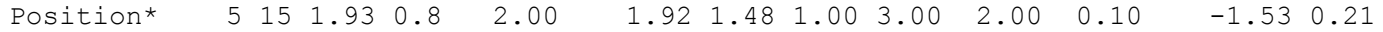

$>$ describe (subset (ds, Group=="Treatment"))

vars $n$ mean sd median trimmed mad min max range skew kurtosis se

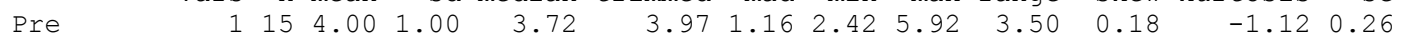

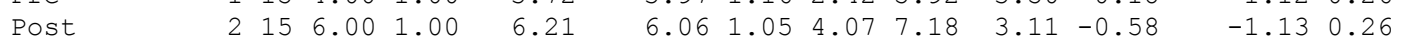

$\begin{array}{lllllllllllll}\text { FollowUp } \quad 3 & 15 & 5.50 & 1.00 & 5.33 & 5.44 & 0.98 & 4.32 & 7.52 & 3.20 & 0.59 & -0.96 & 0.26\end{array}$

$\begin{array}{llllllllllllllll}\text { Group* } & 4 & 15 & 2.00 & 0.00 & 2.00 & 2.00 & 0.00 & 2.00 & 2.00 & 0.00 & \text { NaN } & \text { NaN } & 0.00\end{array}$

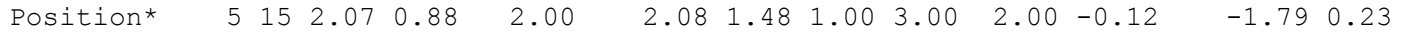

$68>$

$69>\mathrm{ds} 1<-\mathrm{ds}$

$70>$ ds1\$Group<-as.numeric (ds1\$Group) -1 


\section{Nimon et al.}

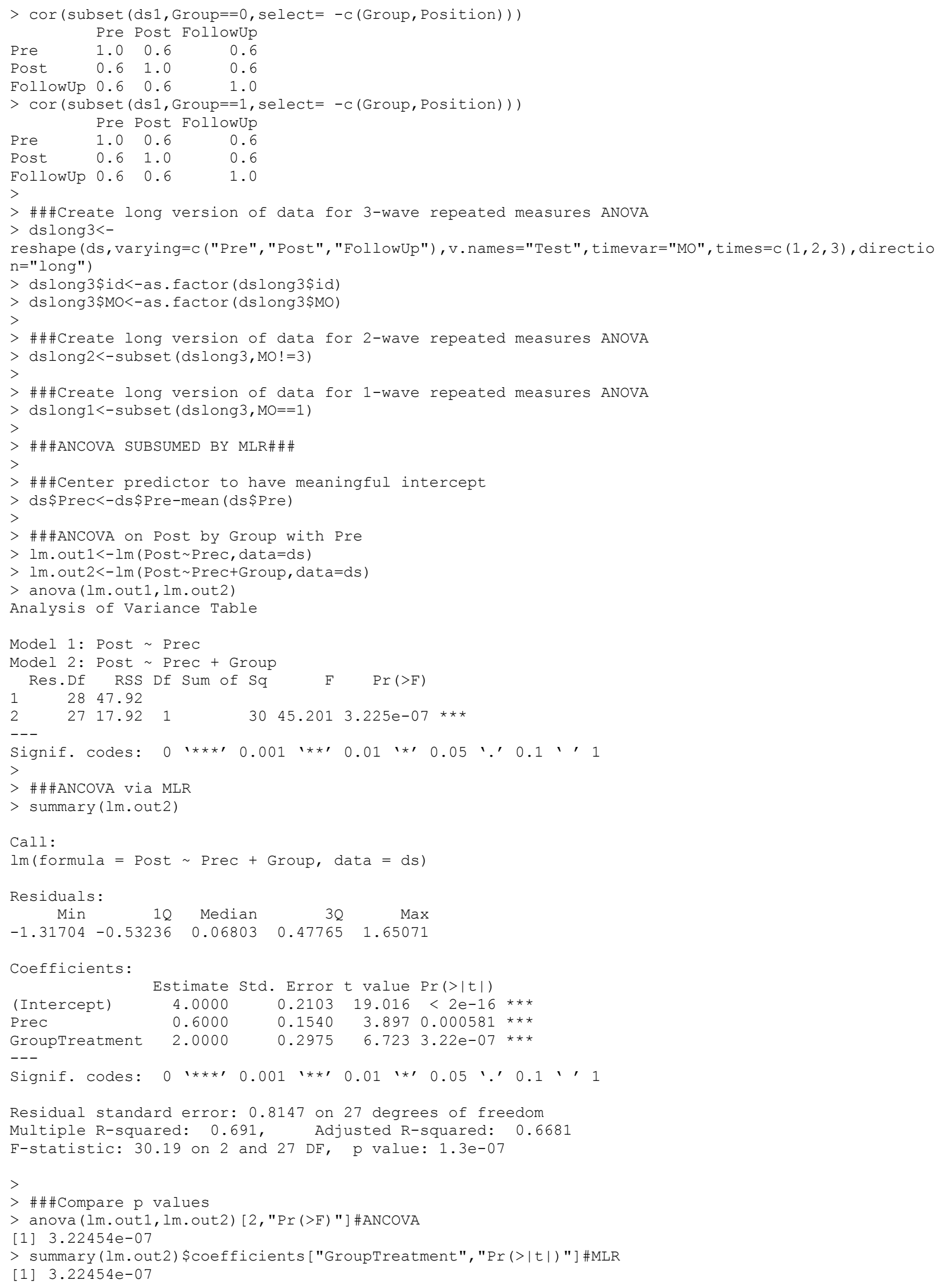


Nimon et al.

213

214

215

216

217

218

219

220

221

222

223

224

225

226

227

228

229

230

231

232

233

234

235

236

237

239

240

241

242

243

244

245

246

247

248

249

250

251

252

253

254

255

256

257

258

259

260

261

262

263

264

265

266

267

268

269

270

271

272

273

274

275

276

277

278

279

280

281

282

283

Residual standard error: 0.8147 on 27 degrees of freedom

Multiple R-squared: 0.691, Adjusted R-squared: 0.6681

F-statistic: 30.19 on 2 and 27 DF, p-value: $1.3 e-07$

$>$ summary(lm.out2) \$coefficients ["(Intercept)","Estimate"]

[1] 4

$>$ summary (lm.out2) \$coefficients [" (Intercept)","Estimate"]+

+ summary (lm.out2) \$coefficients ["GroupTreatment", "Estimate"]

[1] 6

$>$

$>$ \#\#\#ANOVA SUBSUMED BY MLR\#\#\#

$>$ \#\#\#ANOVA on Post by Position

$>$ (aout<-anova (aov (Post Position, data=ds)))

Analysis of Variance Table

Response: Post

Df Sum Sq Mean Sq F value $\operatorname{Pr}(>F)$

$\begin{array}{llllll}\text { Position } 2 & 1.376 & 0.6880 & 0.3281 & 0.7232\end{array}$

Residuals $27 \quad 56.624 \quad 2.0972$

$>$

$>$ \#\#\#MLR on Post by Position using MLR

$>1 \mathrm{~m}$. out $<-1 \mathrm{~m}$ (Post Position, data=ds)

$>\operatorname{summary}(1 \mathrm{~m}$. out $)$

Call:

lm(formula = Post $~$ Position, data $=\mathrm{ds})$

Residuals:

Min $1 Q$ Median $3 Q \quad$ Max

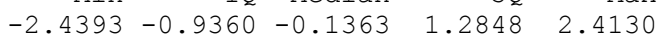

Coefficients:

Estimate std. Error $t$ value $\operatorname{Pr}(>|t|)$

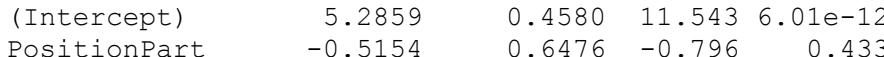

$\begin{array}{lllll}\text { Positionseasonal } & -0.3424 & 0.6476 & -0.529 & 0.601\end{array}$

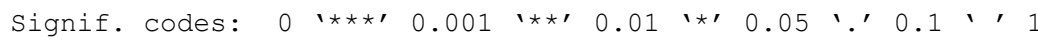

Residual standard error: 1.448 on 27 degrees of freedom

Multiple R-squared: 0.02372, Adjusted R-squared: -0.04859

F-statistic: 0.3281 on 2 and 27 DF, p-value: 0.7232

$>$

$>$ \#\#\#Compare p values

$>$ aout ["Position", "Pr(>F) "] \#ANOVA

[1] 0.7231535

$>$ anova(lm.out) ["Position", "Pr (>F)"] \#MLR

[1] 0.7231535

$>$ \#\#\#Compare test statistics

$>$ aout ["Position", "F value"] \#ANOVA

[1] 0.3280564

$>\operatorname{summary}(\mathrm{lm}$.out) \$fstatistic ["value"] \#MLR value

0.3280564

$>$ \#\#\#Compare effect sizes

$>$ aout ["Position", "Sum Sq"]/sum(aout [,"Sum Sq"]) \#ANOVA

[1] 0.02372397

$>$ summary (lm.out) \$r. squared\#MLR

[1] 0.02372397

$>$

$>$ \#\#\#Compare group means

$>$ \#\#\#ANOVA

$>$ aggregate (ds\$Post ds\$Position, ds, mean)

ds\$Position ds\$Post

$1 \quad$ Full 5.285930

$2 \quad$ Part 4.770533 


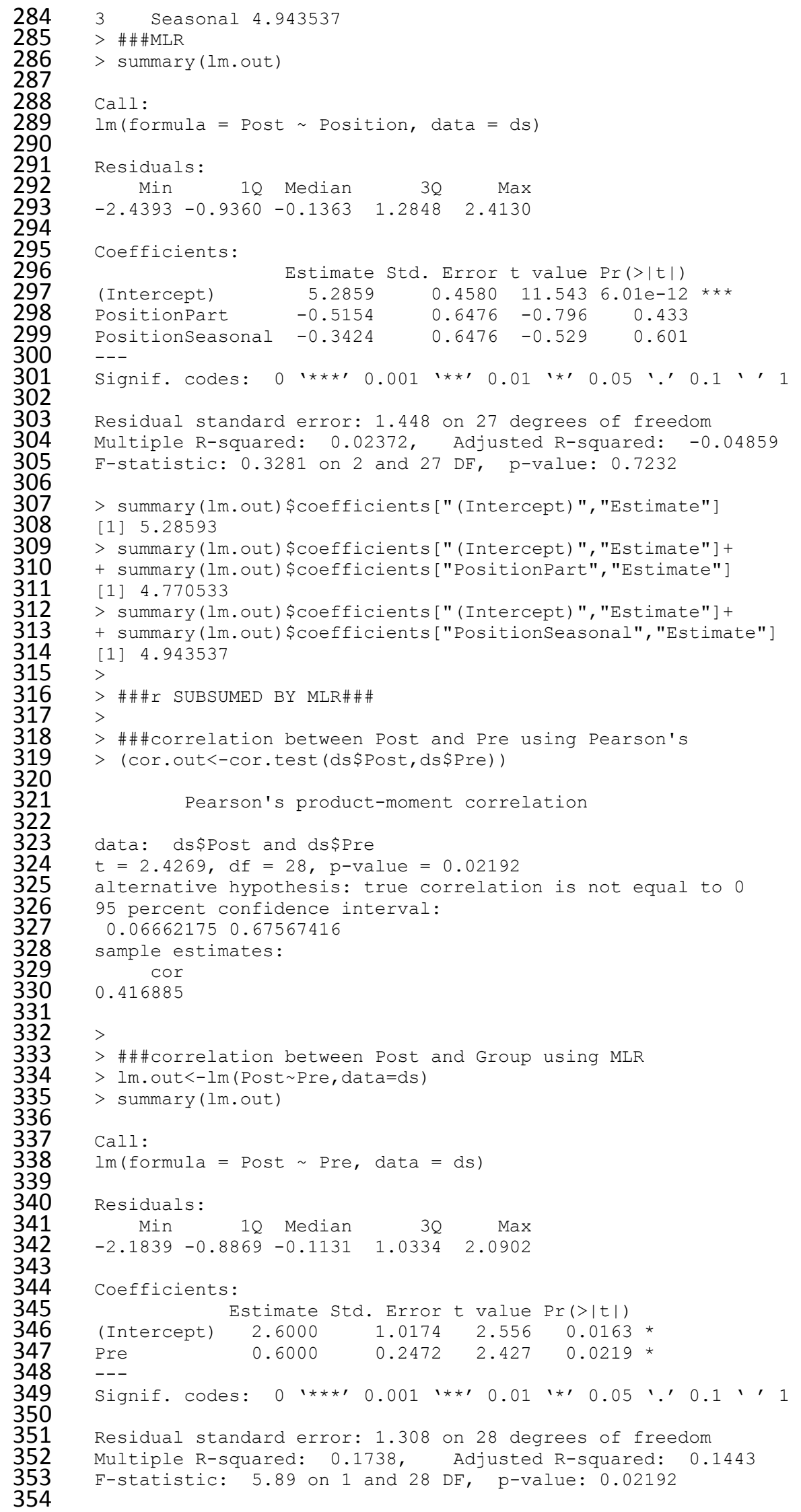




\section{Nimon et al.}

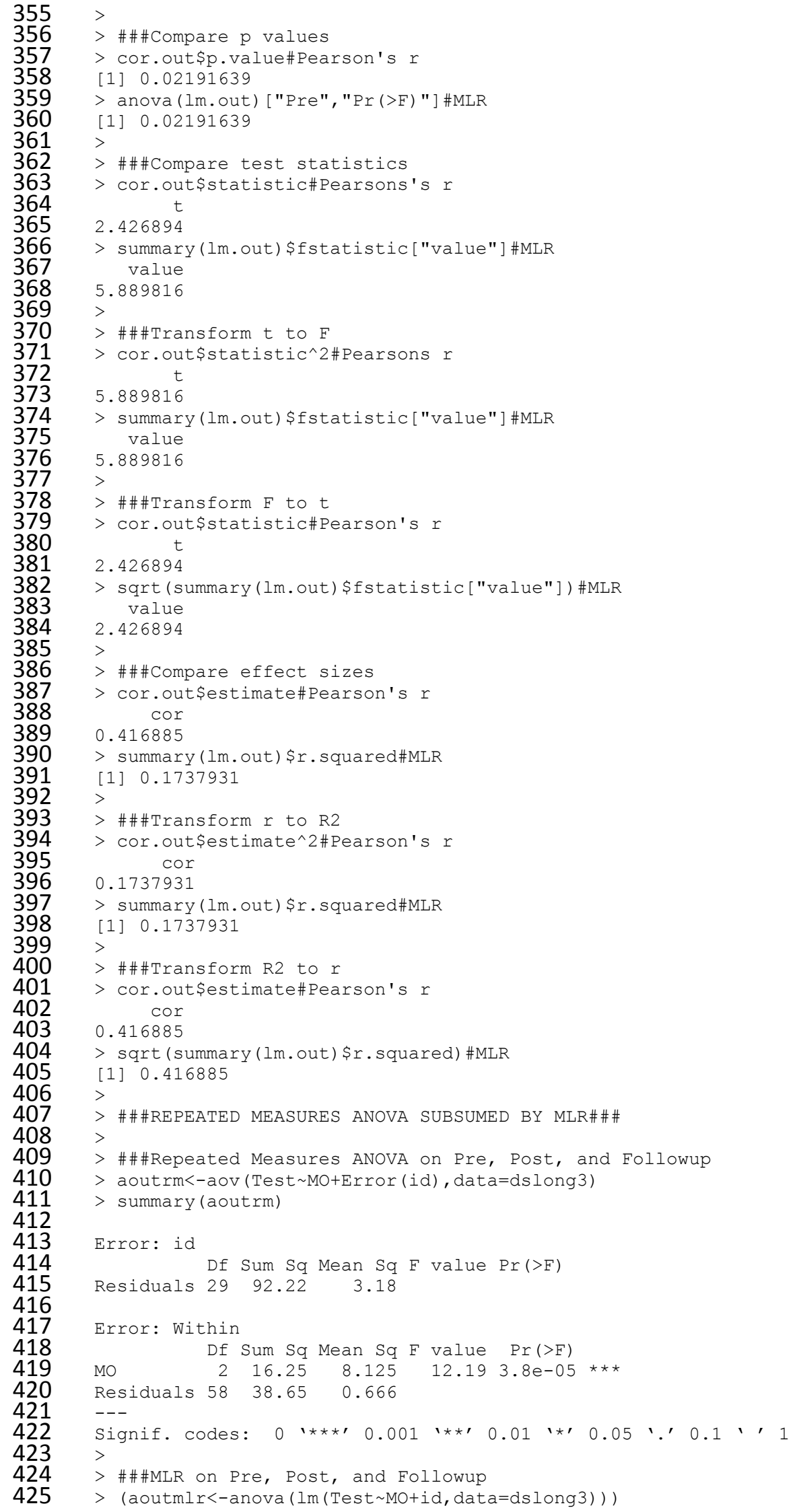




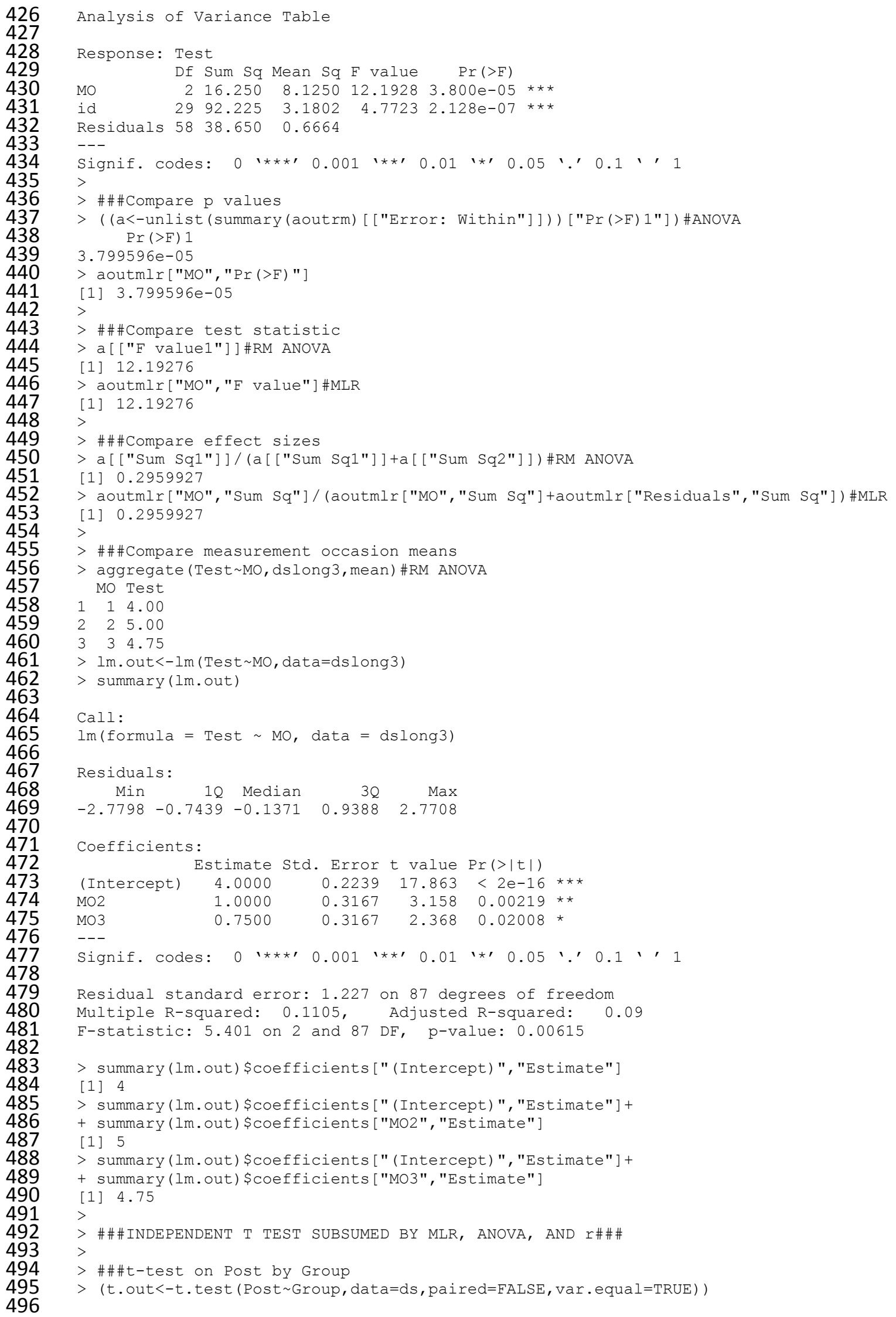




\section{Nimon et al.}

498

499

500

501

502

503

504

505

506

507

508

509

510

511

512

513

514

515

516

517

518

519

520

521

522

523

524

525

526

527

528

529

530

531

532

533

534

535

536

537

538

539

541

542

543

544

545

546

547

548

549

550

551

552

553

554

555

556

557

558

559

560

561

562

563

564

565

566

567

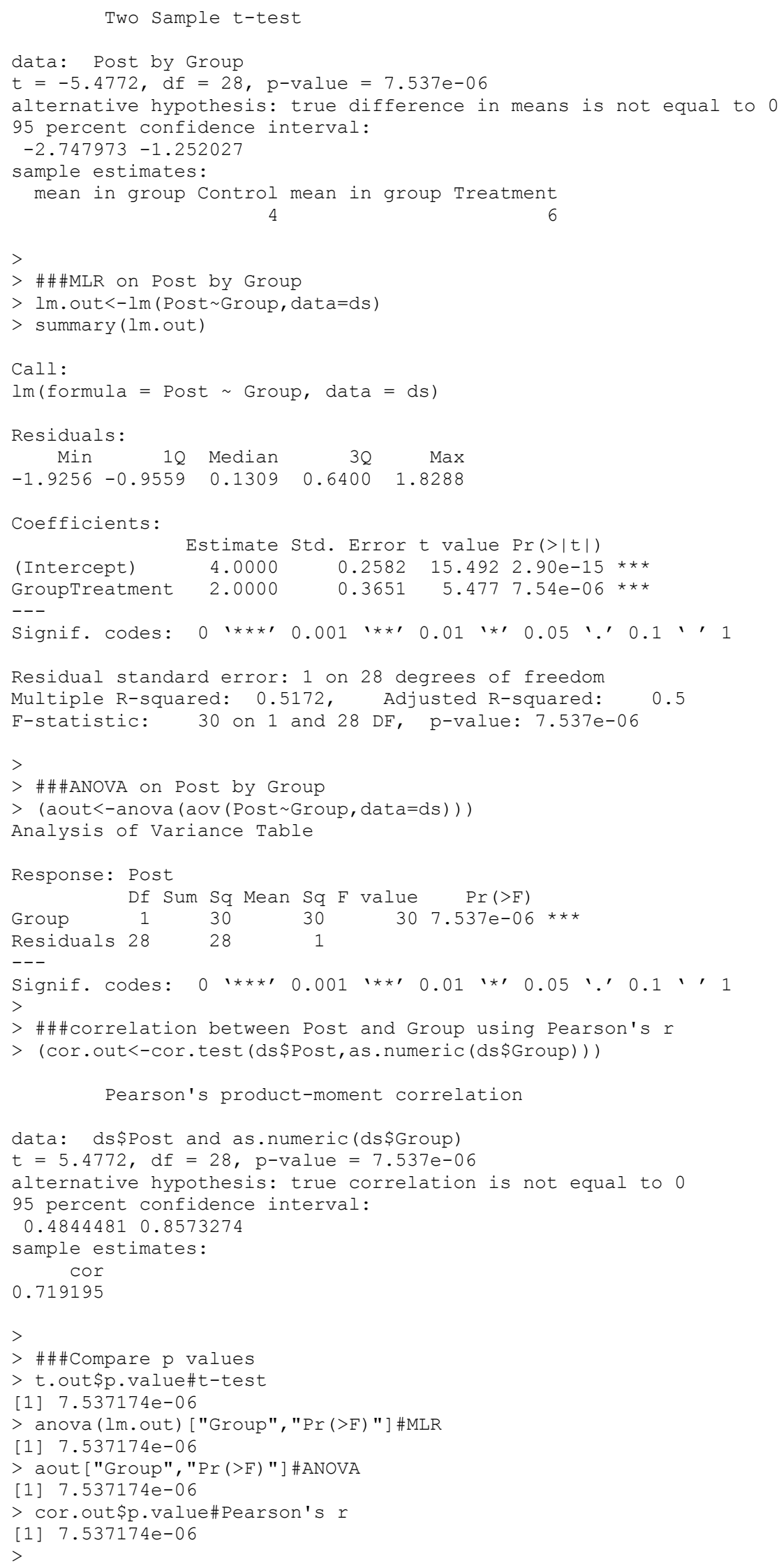




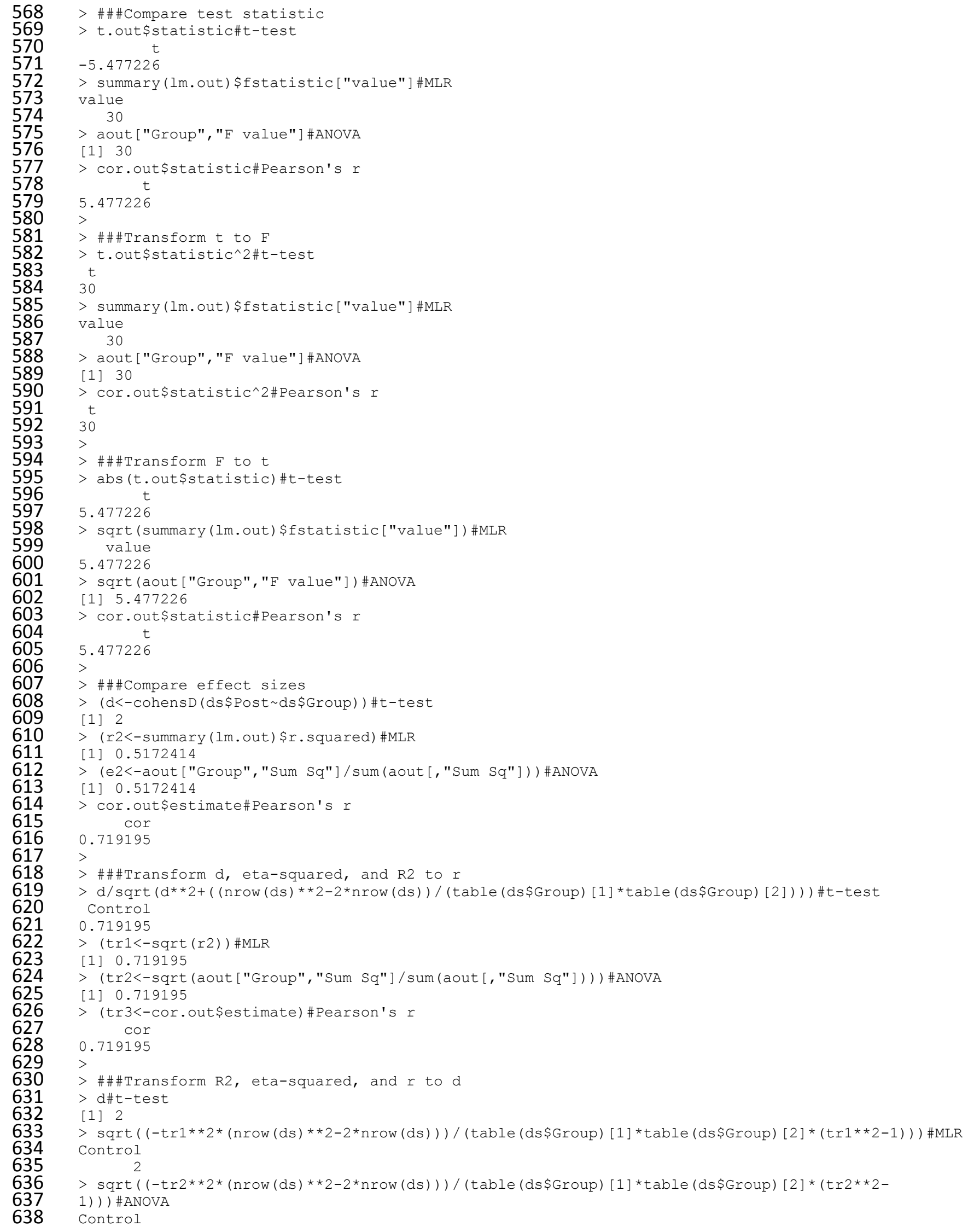




\section{Nimon et al.}

639

640

641

642

643

644

645

646

647

648

649

650

651

652

653

654

655

656

657

658

659

660

661

662

663

664

665

666

667

668

669

670

671

672

673

674

675

676

677

678

679

680

681

682

683

684

685

686

687

688

689

690

691

692

693

694

695

696

697

698

699

700

701

702

703

704

705

706

707

708

709

2

$\operatorname{sqrt}((-\operatorname{tr} 3 * * 2 *(\operatorname{nrow}(d s) * * 2-2 *$ nrow $(d s))) /(\operatorname{table}(d s \$$ Group $)[1] *$ table $(d s \$ G r o u p)[2] *(\operatorname{tr} 3 * * 2-$

1))) \#Pearson's r

cor

2

$>$ \#\#\#Transform $d$ and $r$ to R2/eta-squared

$>(d / \operatorname{sqrt}(d * * 2+(($ nrow $(d s) * * 2-2 *$ nrow $(d s)) /($ table (ds\$Group) [1] *table (ds\$Group) [2] ) ) ) **2\#t-test Control

0.5172414

$>$ r2\#MLR

[1] 0.5172414

$>$ e2\#ANOVA

[1] 0.5172414

$>$ tr $3 * * 2$ \#Pearson's r cor

0.5172414

$>$

$>$ \#\#\#Compare group means

$>$ t.out\#t-test

Two Sample t-test

data: Post by Group

$t=-5.4772, \mathrm{df}=28, \mathrm{p}$-value $=7.537 \mathrm{e}-06$

alternative hypothesis: true difference in means is not equal to 0

95 percent confidence interval:

$-2.747973-1.252027$

sample estimates:

mean in group Control mean in group Treatment

4

$>$ summary (lm.out) \#MLR

Call:

$\operatorname{lm}($ formula $=$ Post $\sim$ Group, data $=d s)$

Residuals:

Min 10 Median $30 \quad$ Max

$\begin{array}{lllll}-1.9256 & -0.9559 & 0.1309 & 0.6400 & 1.8288\end{array}$

Coefficients:

$\begin{array}{lrrrr} & \text { Estimate Std. Error } t \text { value } \operatorname{Pr}(>|t|) \\ \text { (Intercept) } & 4.0000 & 0.2582 & 15.492 & 2.90 e-15 \quad * * * \\ \text { GroupTreatment } & 2.0000 & 0.3651 & 5.477 & 7.54 e-06\end{array}$ ***

$---$

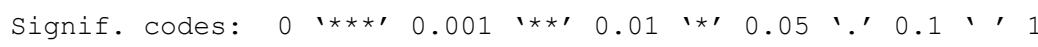

Residual standard error: 1 on 28 degrees of freedom

Multiple R-squared: 0.5172, Adjusted R-squared:

F-statistic: $\quad 30$ on 1 and 28 DF, p-value: $7.537 e-06$

> summary(lm.out) \$coefficients ["(Intercept)", "Estimate"]

[1] 4

> summary (lm.out) \$coefficients ["(Intercept)", "Estimate"]+

+ summary (lm.out) \$coefficients ["GroupTreatment", "Estimate"]

[1] 6

$>$ aggregate (ds\$Post ds\$Group, ds, mean) \#ANOVA

ds\$Group ds\$Post

1 Control

Treatment 6

$>$ aggregate (ds\$Post ds\$Group, ds, mean) \#Pearson's r

ds\$Group ds\$Post

1 Control 4

2 Treatment 6

$>$ \#\#\#PAIRED T TEST SUBSUMED BY MLR and ANOVA\#\#\#

\#\#\#t-test on Pre and Post

$>$ (t.out<-t.test (ds\$Post, ds\$Pre, paired=TRUE)) 
711

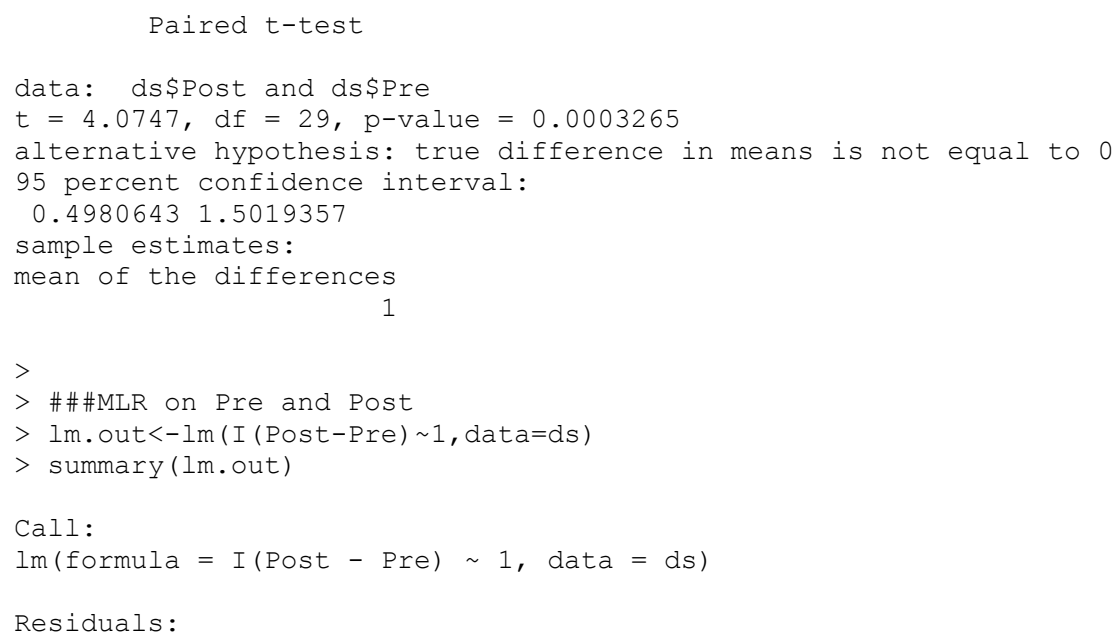




\section{Nimon et al.}

[1] 16.60305

782

$>$ f\#ANOVA

F value1

16.60305

$>$

$>$ \#\#\#Transform $\mathrm{F}$ to $\mathrm{t}$

$>$ t1\#t-test

4.074684

$>$ t2\#MLR

[1] 4.074684

$>$ sqrt (f) \#ANOVA

F value1

4.074684

$>$

$>$ \#\#\#Compare effect sizes

$>$ t $1{ }^{*}$ sqrt $(2 *(1-\operatorname{cor}(d s \$ P o s t, d s \$ P r e)) /$ nrow $(d s))$ \#t-test

$0.8033882^{t}$

$>$ t $2{ }^{*} \operatorname{sqrt}(2 *(1-\operatorname{cor}($ ds $\$$ Post, ds $\$$ Pre $)) /$ nrow $(d s)) \# M L R$

[1] 0.8033882

$>\operatorname{sqrt}(\mathrm{f}){ }^{*} \operatorname{sqrt}(2 *(1-\operatorname{cor}(\mathrm{ds} \$$ Post, ds\$Pre $)) /$ nrow $(\mathrm{ds}))$ \#MLR

$\mathrm{F}$ valuel

0.8033882

$>$

$>$ \#\#\#Compare group means

$>$ t.out\$estimate\#t-test

mean of the differences

summary (lm.out) \$coefficients [" (Intercept)", "Estimate"] \#MLR

[1] 1

$>$ describe (ds\$Post-ds\$Pre) \$mean\#ANOVA

[1] 1

$>$

$>$ \#\#\#SINGLE SAMPLE T TEST SUBSUMED BY MLR\#\#\#

$>$

$>$ \#\#\#t-test on Pre

$>(t$. out $<-t$. test $($ ds $\$$ Pre $))$

One Sample t-test

data: ds\$Pre

$t=22.2967, \mathrm{df}=29, \mathrm{p}$-value $<2.2 \mathrm{e}-16$

alternative hypothesis: true mean is not equal to 0

95 percent confidence interval:

$3.633088 \quad 4.366912$

sample estimates:

mean of $\mathrm{x}$

4

$>$

$>$ \#\#\#MLR on on Pre

$>\operatorname{lm}$.out $<-\operatorname{lm}($ Pre 1 , data $=\mathrm{ds})$

$>\operatorname{summary}(\mathrm{lm}$. out $)$

Call:

$\operatorname{lm}($ formula $=$ Pre $\sim 1$, data $=d s$ )

Residuals:

Min 1Q Median 3Q Max

$\begin{array}{llllll}-2.7328 & -0.4991 & -0.1004 & 0.6342 & 1.9180\end{array}$

Coefficients:

Estimate std. Error t value $\operatorname{Pr}(>|t|)$

(Intercept) $4.0000 \quad 0.1794 \quad 22.3 \quad<2 e-16 * \star *$

Signif. codes: 0 、*** 0.001 、*' 0.01 、* 0.05 ', 0.1 ', 1

Residual standard error: 0.9826 on 29 degrees of freedom

850

851

$>$ 


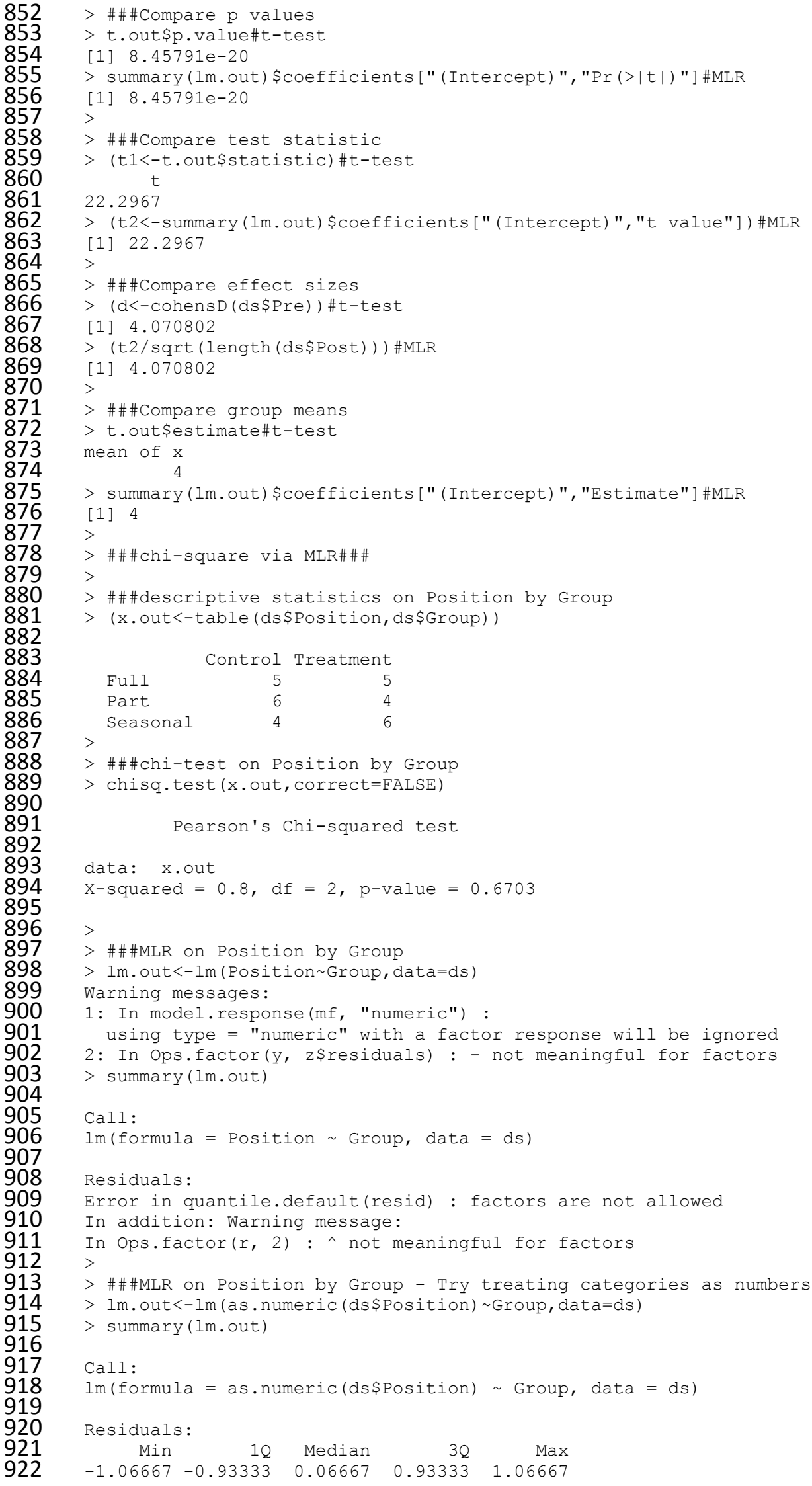


Nimon et al.

923

924

925

926

927

928

929

930

931

932

933

934

935

936

937

938

939

940

941

942

943

944

945

946

947

948

949

950

951

952

953

954

955

956

957

958

959

960

961

962

963

964

965

966

967

968

969

970

971

972

973

974

975

976

977

978

979

980

981

982

983

984

985

986

987

988

989

990

991

992

993

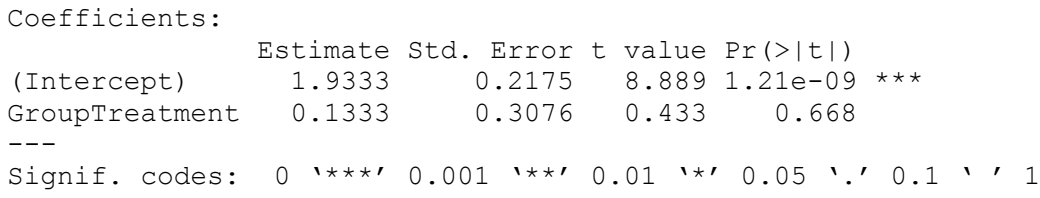




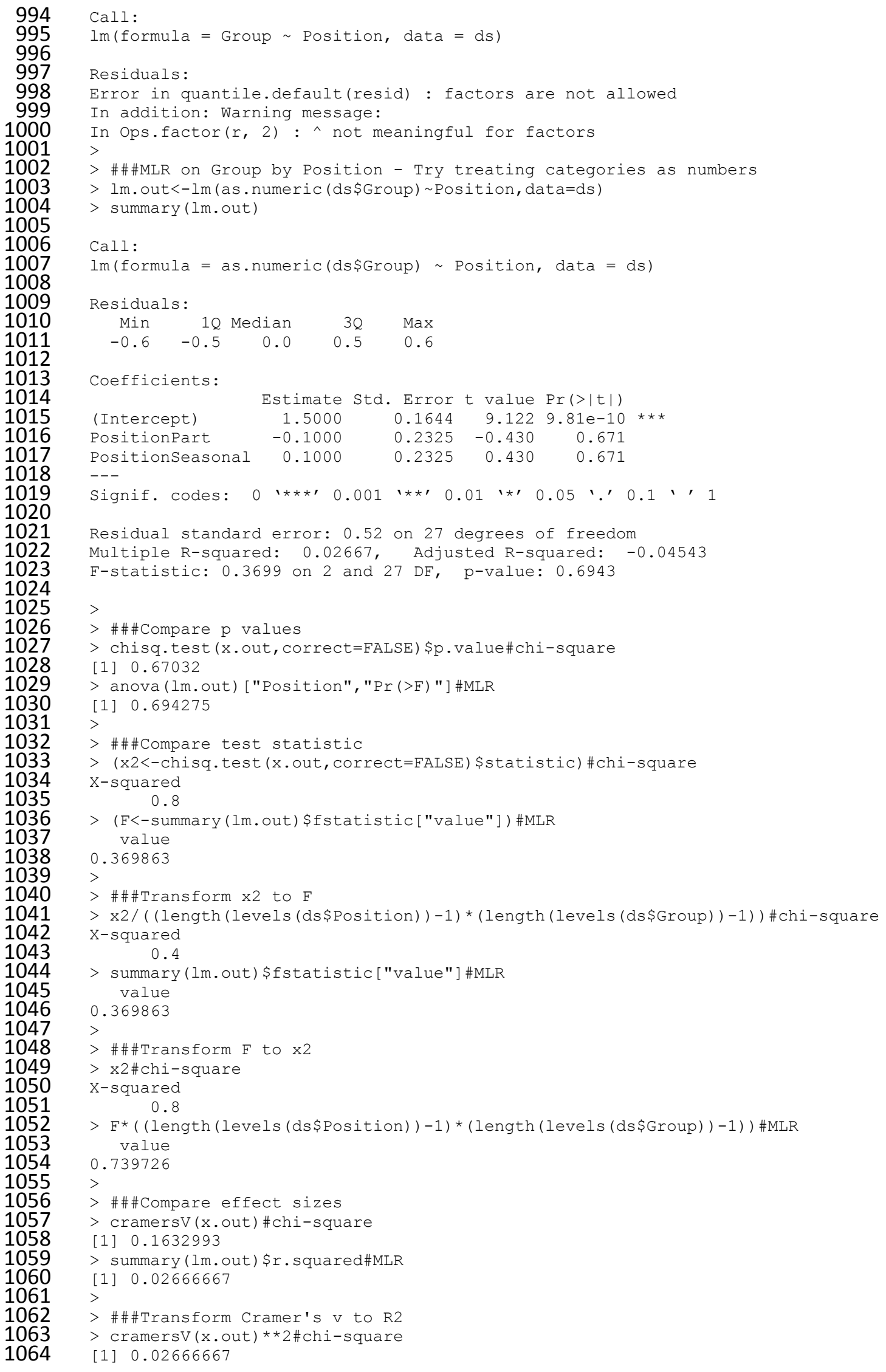


Nimon et al.

1065

1066

1067

$>$ summary (lm.out) \$r.squared\#MLR

[1] 0.02666667

1068

$>$

$>$ \#\#\#Transform R2 to Cramer's v

$1069>$ cramersv (x.out) \#chi-square

1070 [1] 0.1632993

$1071>$ sqrt (summary (lm.out) \$r.squared) \#MLR

1072

[1] 0.1632993

1073

$>$ 